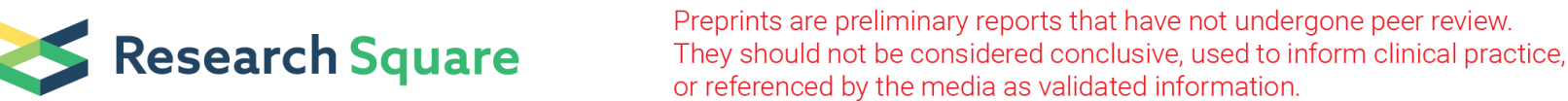

\section{Three different genetic risk scores based on Fatty Liver Index, Magnetic Resonance Imaging and lipidomic for a nutrigenetic personalized management of NAFLD: the Fatty Liver in Obesity study}

\section{Nuria Pérez-Díaz-del-Campo}

University of Navarre Faculty of Pharmacy and Nutrition: Universidad de Navarra Facultad de Farmacia y Nutricion

\section{Jose I. Riezu-Boj}

University of Navarra: Universidad de Navarra

\section{Bertha Araceli Marin-Alejandre}

University of Navarre Faculty of Pharmacy and Nutrition: Universidad de Navarra Facultad de Farmacia y Nutricion

\section{J. Ignacio Monreal}

Clínica Universidad de Navarra: Clinica Universidad de Navarra

Mariana Elorz

Clínica Universidad de Navarra: Clinica Universidad de Navarra José Ignacio Herrero

Clínica Universidad de Navarra: Clinica Universidad de Navarra

\section{Alberto Benito-Boillos}

Clínica Universidad de Navarra: Clinica Universidad de Navarra

\section{Fermín I. Milagro}

University of Navarre Faculty of Pharmacy and Nutrition: Universidad de Navarra Facultad de Farmacia y Nutricion

\section{Josep A. Tur}

University of the Balearic Islands: Universitat de les Illes Balears

Itziar Abete ( $\nabla$ iabetego@unav.es )

University of Navarra https://orcid.org/0000-0003-0479-8825

\section{Angeles Zulet}

University of Navarre Faculty of Pharmacy and Nutrition: Universidad de Navarra Facultad de Farmacia y Nutricion

\section{J. Alfredo Martinez}


University of Navarre Faculty of Pharmacy and Nutrition: Universidad de Navarra Facultad de Farmacia y Nutricion

\section{Research Article}

Keywords: NAFLD, Genetic risk score, Fatty Liver Index, Lipidomic, Magnetic Resonance Imaging

Posted Date: March 22nd, 2021

DOI: https://doi.org/10.21203/rs.3.rs-333145/v1

License: (c) (1) This work is licensed under a Creative Commons Attribution 4.0 International License. Read Full License 


\section{Abstract \\ Background}

Non-alcoholic fatty liver disease (NAFLD) affects $25 \%$ of the global population. The pathogenesis of NAFLD is complex where available data unveils that genetics and ascribed interactions with environmental factors may play an important role in the development of this morbid condition. The purpose of this investigation was to assess genetic and non-genetic determinants putatively involved in the onset and progression of NAFLD after a 6-months weight-loss nutritional treatment.

\section{Methods}

A group of 86 overweight/obese subjects with NAFLD from the Fatty Liver in Obesity (FLiO) study were enrolled and metabolically evaluated at baseline and after 6-months. Also, a total of 95 single nucleotide polymorphisms (SNPs) related to obesity and weight loss were analyzed by a targeted next-generation sequencing system. Three genetic risk scores (GRS) concerning the improvement on hepatic health evaluated by minimally invasive methods such as the Fatty Liver Index (FLI) $\left(\mathrm{GRS}_{\mathrm{FLI}}\right)$, lipidomicOWLiver ${ }^{\circledR}$-test (GRS $\mathrm{OWL}_{\mathrm{L}}$ ) and Magnetic Resonance Imaging (MRI) (GRS $\mathrm{MRI}$ ), were derived by adding the risk alleles genotypes. Body composition, liver injury-related markers and dietary intake were also monitored.

\section{Results}

Overall, 26 SNPs were independently associated with the change on FLI, 16 SNPs with OWLiver®-test and 8 SNPs with MRI, which were specific for every diagnosis tool. After adjusting for gender, age and other related predictors (insulin resistance, inflammatory biomarkers and dietary intake at baseline) the calculated $\mathrm{GRS}_{\mathrm{FL}}, \mathrm{GRS}_{\mathrm{OWL}}$ and $\mathrm{GRS}_{\mathrm{MRI}}$ were major contributors of the improvement on hepatic status. Thus, fitted linear regression models showed a variance of $53 \%\left(\operatorname{adj} . R^{2}=0.53\right)$ in hepatic functionality (FLI), $16 \%$ (adj. $R^{2}=0.16$ ) in lipidomic metabolism (OWLiver ${ }^{\circledR}$-test) and $34 \%$ (adj. $R^{2}=0.34$ ) in liver fat content (MRI).

\section{Conclusion}

These results demonstrate that three different genetic scores can be useful for the personalized management of NAFLD, whose treatment must rely on specific dietary recommendations guided by the measurement of specific genetic biomarkers.

\section{Trial registration:}


The FLiO study: Fatty Liver in Obesity study, NCT03183193. Registered 12 June 2017 - Retrospectively registered, https://clinicaltrials.gov/ct2/show/NCT03183193

\section{Background}

Non-alcoholic fatty liver disease (NAFLD) is the leading cause of liver disease in high-income countries, affecting more than $25 \%$ of the population [1]. NAFLD is defined as fatty liver without injury of the hepatocyte in the form of ballooning, with or without inflammation $[2,3]$ and it has become one of the most common causes of chronic liver diseases [3].

The NAFLD etiology is multifactorial and yet incompletely understood, but ultimately appears as determined by the combination of environmental factors, such as excessive adiposity or the presence of type 2 diabetes (T2D) as well as the accumulation of intrahepatic lipids, alterations of energy metabolism, insulin resistance and inflammatory processes, where the genetic make-up may emerge [1, 4]. Other factors such as obesity and a sedentary lifestyle, together with metabolic syndrome features and ethnicity, influence the risk of NAFLD [5].

The gold standard for the diagnosis of NAFLD is liver biopsy [6]. However, the decision about when to perform this screening remains controversial [2], being necessary the search for less invasive methods for screening patients suspected of this disease [6-9]. To date, ultrasonography is recommended as the firstline diagnostic method in assessing steatosis [6]. Moreover, magnetic resonance imaging (MRI) has also shown a high accuracy for diagnosing liver fat content [10], as well as advances in the analysis of big data from lipidomic have provided novel insights [11]. Furthermore, non-invasive biomarkers and some validated algorithms such as the Fatty Liver Index (FLI) have also become a useful tool for the diagnosis of simple steatosis and hepatic functionality [12].

Heritability and family history have a clinically relevant impact on fatty liver disease onset and progression $[1,5,13]$. In particular, the genetic variants in the genes PNPLA3 (rs738409), TM6SF2 (rs58542926), GCKR (rs1260326) and MBOAT7 (rs641738) have been associated with the risk of NAFLD [14-16]. Not only these genes, but also other genetic variants related with obesity traits and loci have been associated with a higher risk of developing a severe stage of NAFLD $[13,17]$.

Although these polymorphisms explain only a small fraction of the total heritability of NAFLD, it is possible that the combination of specific single nucleotide polymorphisms (SNPs) into a Genetic Risk Score (GRS) could increase the detection and evolution of NAFLD $[5,10,18]$. Hence, some models have investigated the impact of the genetic predisposition to accumulate liver fat on NAFLD. In this context, some researchers have demonstrated an association between the combination of SNP in a GRS with de novo hepatocellular carcinoma (HCC) $[15,19]$, but also with higher hepatic fat content, total cholesterol, steatosis degree and alanine aminotransferase (ALT) levels $[5,14,16]$. In this sense, it has been suggested an independent regulation of fat distribution from total adiposity where genes near loci regulating total body mass are enriched for expression in the Central Nervous System, while genes for fat distribution are enriched in adipose tissue itself [20]. 
Concerning treatment, there are no specific medications that directly treat NAFLD [3, 21], being lifestyle modifications and weight control the most fundamental steps in the management of NAFLD [10]. In this context, the European Association for the Study of the Liver (EASL) recommends diet and physical activity as the best treatment for steatosis [22-24]. However, increasing evidence suggests that interindividual variability in weight loss also depends upon interactions between genetic and environmental factors, including lifestyle [25-27]. Besides, the well and precise characterization of each gene and its different related pathways is essential in order to devise new therapies. Indeed, a personalized treatment taking into account genetics, lifestyle and specific macronutrient recommendations is needed $[24,28,29]$.

In this sense, the aim of the present study was to assess genetic and non-genetic factors putatively involved in the improvement of the hepatic health after a 6-month hypocaloric nutritional treatment. To test this hypothesis, we combined 38 obesity-related genetic variants associated with different NAFLD non-invasive methods based on the change of hepatic functionality (FLI), lipid metabolism (OWLiver®test) and liver fat content (by MRI) into three differences scores, where the role of baseline status was assessed to predict outcomes from a precision nutrition management.

\section{Material And Methods 2.1 Study design}

The current randomized controlled trial was designed to compare the effectiveness of two weight loss dietary strategies with different nutritional features, anthropometric measurements, body composition and biochemical markers on hepatic health, in overweight or obese subjects with ultrasonography-proven liver steatosis, as described elsewhere [30,31]. The intervention had a duration of 24-months and the participants were randomly assigned to the American Heart Association (AHA) or the Fatty Liver in Obesity (FLiO) group [32]. However, the present study was performed concerning the results after 6 months of follow-up. The study was approved by the Research Ethics Committee of the University of Navarra (ref. 54/2015) and registered at www.clinicaltrials.gov (FLiO: Fatty Liver in Obesity study; NCT03183193). Each subject gave written informed consent prior to enrollment in the study. All the procedures were performed in accordance with the Declaration of Helsinki and the study was conducted following the CONSORT 2010 guidelines.

\subsection{Study participants}

A total of 98 men and women with overweight or obesity (Body Mass Index (BMI) $\geq 27.5 \mathrm{~kg} / \mathrm{m}^{2}$ to $<40$ $\mathrm{kg} / \mathrm{m}^{2}$ ) between $40-80$ years old and with hepatic steatosis confirmed by abdominal ultrasonography fulfilled the selection criteria and were enrolled in the study [33]. After 6 months a total of 76 participants completed the evaluation. Exclusion criteria included the presence of known liver disease other than NAFLD, $\geq 3 \mathrm{~kg}$ of body weight loss in the last 3 months, excessive alcohol consumption ( $>21$ standard drinks per week in men and > 14 standard drinks per week for women) [34], endocrine disorders 
(hyperthyroidism or uncontrolled hypothyroidism), pharmacological treatments (immunosuppressants, cytotoxic agents, systemic corticosteroids or other drugs that could potentially cause hepatic steatosis or altering liver tests [35], active autoimmune diseases or requiring pharmacological treatment, the use of weight modifiers and severe psychiatric disorders, and the lack of autonomy or an inability to follow the diet, as well as the use of weight modifiers, severe psychiatric disorders, and difficulties in following the scheduled visits.

\subsection{Dietary and lifestyle intervention}

Two energy-restricted diets (AHA vs FLiO) were prescribed and compared [31]. Both diets applied an energy restriction of $30 \%$ of the total energy requirements of each participant in order to achieve a loss of at least $3-5 \%$ of the initial body weight, in accordance with the recommendations of the American Association for the Study of Liver Diseases guidelines (AASLD) [35]. After 6 months, both groups achieved comparable results in the evaluated variables and no significant differences in the changes between the intervention groups were found, as reported elsewhere [31]. Therefore, participants were merged and compared together. The habitual dietary intake was registered with a validated semiquantitative food frequency questionnaire (FFQ) of 137-items both at baseline and after the 6-months intervention [36]. The composition of the food items was derived from accepted Spanish food composition tables as previously described [4,37]. The adherence to the Mediterranean Diet was assessed with a validated 17-point score questionnaire [38,39]. A physical activity prescription of 10,000 steps/day was given to the participants $[40,41]$. The physical activity level was evaluated using the validated Spanish version of the Minnesota Leisure-Time Physical Activity Questionnaire [41]. The volume of activity was indicated in metabolic equivalent of the task (METs), as described elsewhere [40].

\subsection{Anthropometric, body composition and biochemical assessments}

Anthropometric variables (body weight, height and waist circumference) and body composition (Lunar iDXA, encore 14.5, Madison, WI, USA) were assessed in fasting conditions at the Metabolic Unit of the University of Navarra following standardized procedures [42]. BMI was calculated as the body weight divided by the squared height $\left(\mathrm{kg} / \mathrm{m}^{2}\right)$. Blood samples were properly collected after overnight fasting of 8-10 hours and processed at the Laboratory of Biochemistry of the University of Navarra Clinic (CUN, Pamplona, Spain). Blood glucose, triglycerides (TG), aspartate aminotransferase (AST), alanine aminotransferase (ALT) and gamma-glutamyltransferase (GGT) concentrations were determined on a Cobas 8000 autoanalyzer with specific commercial kits and following the instructions of the company (Roche Diagnostics, Basel, Switzerland). Insulin, fibroblast growth factor 21 (FGF-21), leptin and adiponectin concentrations were quantified with specific ELISA kits (Demeditec; Kiel-Wellsee, Germany) in a Triturus autoanalyzer (Grifols, Barcelona, Spain). Insulin resistance was estimated using the Homeostasis Model Assessment Index (HOMA-IR), which was computed as HOMA-IR $=$ (insulin $(\mu \mathrm{U} / \mathrm{mL})$ $x$ glucose $(\mathrm{mmol} / \mathrm{L})) / 22.5$ [4]. The Triglycerides/Glucose index (TyG) $(\operatorname{In}[$ triglycerides $(\mathrm{mg} / \mathrm{dL}) \mathrm{x}$ glucose(mg/dL)/2)]) was also calculated as a surrogate of glucose tolerance [43]. 


\subsection{Imaging techniques for the assessment of liver status}

The whole liver evaluation was performed under fasting conditions at the University of Navarra Clinic. Liver steatosis was determined by ultrasonography (Siemens ACUSON S2000 and S3000, Erlangen, Germany) in accordance with previously described methodology [44]. The clinical classification was done according to a 4-point scale: less than 5\% (grade 0), 5-33\% (grade 1), 33-66\% (grade 2), and greater than $66 \%$ (grade 3), as described elsewhere [45]. Liver fibrosis was assessed by transient elastography thought FibroScan ${ }^{\circledR}$ following manufacturer's instructions (Echosens, Paris, France) [46]. Finally, Magnetic Resonance Imaging (Siemens Aera 1,5 T) was used to determine the hepatic volume and the fat and iron content of the liver (Dixon technique) as reported by the manufacturer [30].

Fatty Liver Index (FLI) was calculated using serum triglycerides, BMI, waist circumference, and GGT concentrations using the formula described elsewhere [12].

\subsection{Metabolomics}

The metabolomic test OWLiver ${ }^{\circledR}$ (One Way Liver S.L. Bilbao, Spain) is a fasting blood probe able to measure the degree of NAFLD development [30]. The test score is based on a prospective study, where subjects had previously been diagnosed by liver biopsy [47]. The methodology of this test consisted in the measure of a panel of biomarkers that belong to the family of triacylglycerols (TGs), which are a reflection of the amount of fat and inflammation of the liver [30]. The final OWLiver ${ }^{\circledR}$ score is generated by the relative metabolite concentrations, which are analyzed together in a specific algorithm that gives the probabilities of normal liver, steatosis or NASH.

\subsection{SNP Selection and Genotyping}

A total of 95 genetic variants related to obesity and weight loss were analyzed after an exhaustive bibliographic review following PRISMA criteria [10, 48-50]. More information about these obesity-related SNPs can be found in a previous reports [26]. A total of 86 buccal samples were collected with a liquidbased kit (ORAcollect-DNA, OCR-100, DNA genotek Inc, Ottawa, Canada). Genomic DNA was isolated using the Maxwell ${ }^{\circledR} 16$ Buccal Swab LEV DNA Purification Kit (Promega Corp, Madison, WI, USA). Genotyping was performed by targeted next generation sequencing on lon Torrent PGM equipment (Thermo Fisher Scientific Inc., Waltham, MA, USA)[51], as published [48, 52]. Overall, the amplicon mean size was $185 \mathrm{bp}$. Library construction was carried out using a custom-designed panel and the lon AmpliSeq Library Kit 2.0 (Thermo Fisher Scientific) as per the manufacturer's protocol. The raw data were processed with the lon Torrent Suite Server Version 5.0.4 (Thermo Fisher Scientific Inc, Waltham, MA, USA) using Homo sapiens (genome assembly $\mathrm{Hg} \mathrm{19)}$ as the reference genome for the alignment. A custom-designed Bed file was used to locate the SNPs of interest. Genetic variants were identified with the Torrent Variant Caller 5.0 (Thermo Fisher Scientific) with a minimum coverage value of 20. HardyWeinberg equilibrium, linkage disequilibrium, and haplotype inferences were estimated using the Convert program (Version 1.31) and the Arlequin software (Version 3.0). Hardy Weinberg equilibrium was calculated with a statistical test (Chi-square). 


\subsection{Genetic Risk Score (GRS)}

Once the 95 SNPs were genotyped, three individual GRS were calculated for the change of each noninvasive diagnostic method (FLI, MRI and OWLiver®-test) according to the following steps. Firstly, Kruskal-Wallis tests were performed to identify SNPs statistically or marginally associated with the change on FLI, liver fat content by MRI, and metabolomics assessed by OWLiver ${ }^{\circledR}$-test (absence of allele, presence of one allele or presence of two alleles) in our samples, obtaining a total of 38 SNPs with a $p$ value lower than 0.20 . Secondly, post hoc tests (Mann-Whitney $U$ test pairwise) were run to define differences between genotypes in order to be differentially coded as risk and non-risk groups with these 38 SNPs. A risk genotype was defined as the one that was associated with a lower change of FLI, liver fat content (MRI) and OWLiver ${ }^{\circledR}$-test. Genotypes with similar effects were clustered in a single category. In a third step, Mann-Whitney U test was applied to confirm statistical differences between the categorized genotype groups (risk vs. non-risk), selecting those SNPs showing at least a marginal statistical trend ( $p$ $<0.10)$ and excluding those with low sample $(<10 \%)$ in either category or due to collinearity. To evaluate the combined effects of the previously selected SNPs on the change of FLI, fat liver content and OWLiver ${ }^{\circledR}$-test, the three individual GRS were calculated by summing the number of risk alleles at each locus $[53,54]$.

\subsection{Statistical analysis}

The primary outcome of the study was the weight loss, according to the current recommendations of the AASLD to ameliorate NAFLD features [35]. The sample size was estimated assuming a mean difference of weight-loss of $1.0(1.5 \mathrm{~kg}$ ) between both dietary groups (AHA vs FLiO) with a 95\% confidence interval $(a=0.05)$ and a statistical power of $80 \%(\beta=0.80)$. Considering a dropout rate of $20-30 \%, 50$ subjects were included in each group of the study, even though two subjects were excluded from AHA group due to the presence of important biochemical alterations in the initial assessment. This trial started with 98 participants but only 86 epithelium buccal cells from volunteers were available. Moreover, after 6 months a total of 70 participants had complete information and epithelium buccal cells to carry out the study.

Results with normal distribution were expressed as means \pm standard deviations (SD), whereas continuous skewed variables were presented as medians and interquartile ranges (IQR). Moreover, qualitative variables were expressed as number ( $n$ ) and percentages (\%). The normality of the distribution was checked through Shapiro-Wilk and Kolmogorov-Smirnov test. Statistical differences for continuous variables at baseline (between men and women and according to age) and after the 6-month dietary intervention were estimated using Student's t-tests of independent samples and Wilcoxon-Mann Whitney (for non-normally distributed variables). Categorical variables were compared using a Chi-squared test.

Diagnostic tests of the regression assumption for linearity and equal variance of residuals, and the variance inflation factor (VIF) for testing collinearity between independent variables were conducted. Multiple linear regression models were used to predict FLI, liver fat content (by MRI) and OWLiverß-test changes. All the designed GRS were used as continues variables in the multiple linear regression models. In addition to genetic variants, other conventional factors of personalization were evaluated, including 
age, sex, and the following variables at baseline: insulin (U/mL), FGF-21 (pg/ml) and protein (\%), as well as potential interaction introducing the corresponding interaction terms inro the models, were investigated.

All $p$-values presented are two-tailed and were considered statistically significant at $p<0.05$. Analyses were carried out using Stata version 12.0 software (StataCorp, College Station, TX, USA http://www.stata.com).

\section{Results}

Baseline characteristics of the participants, including body composition, biochemical, and nutritional characteristics, are reported separated by sex and age (Table 1). Overall, $57 \%(n=49)$ of subjects were men. The average values of weight, waist circumference followed expected trends depending on sex. Triglycerides and insulin resistance-related variables (HOMA-IR and TyG) showed statistical differences between genders, being higher in men as compared to women. However, leptin concentration was significantly lower in males $(20.1 \mathrm{ng} / \mathrm{mL}$ vs. $46.0 \mathrm{ng} / \mathrm{mL}$ in females). Analyzing variables associated with liver injury, statistical differences were observed in the fatty liver index (76.0 vs 89.6) and in liver fat content measured by MRI (4.5\% vs. $6.5 \%)$, showing worst hepatic health values in men in both measures. On the other hand, lipidomic analysis (OWLiver ${ }^{\circledR}$-test) did not show significant differences. Concerning results according to age significant differences were only observed on weight, glucose and adiponectin concentrations and MedDiet Score. Regarding dietary, the nutritional pattern of the study population was characterized by a relatively high consumption of energy derived from fat (37.4\%), a concomitant low intake of carbohydrates (42.8\%) and an average protein intake of $16.8 \%$. Moreover, significant improvements in body composition, biochemical parameters, hepatic health variables, dietary intake and lifestyle factors were observed after the 6-months nutritional intervention, following the expected trends. 
Table 1

Baseline characteristics and after 6 months of dietary intervention and according to sex and age Baseline

\begin{tabular}{|c|c|c|c|c|c|c|}
\hline & $\begin{array}{l}\text { All } \\
\text { participants }\end{array}$ & Women & Men & $\leq 50 y$ & $>50 y$ & 6-months ${ }^{a}$ \\
\hline$n$ & 86 & 37 & 49 & 43 & 43 & 70 \\
\hline \multicolumn{7}{|l|}{$\begin{array}{l}\text { Body } \\
\text { composition }\end{array}$} \\
\hline Weight (kg) & $95.0(13.9)$ & $\begin{array}{l}88.1 \\
(13.0)\end{array}$ & $\begin{array}{l}100.3 \\
(12.3) \star \star \star\end{array}$ & $\begin{array}{l}98.5 \\
(13.8)\end{array}$ & $\begin{array}{l}91.6(13.3) \\
*\end{array}$ & $\begin{array}{l}84.4(75.1 ; \\
92.1)) * \star \star\end{array}$ \\
\hline BMI $\left(\mathrm{kg} / \mathrm{m}^{2}\right)$ & $\begin{array}{l}32.8(30.6 \\
35.8)\end{array}$ & $\begin{array}{l}32.9 \\
(30.0 \\
36.2)\end{array}$ & $\begin{array}{l}32.5(30.9 \\
35.8)\end{array}$ & $\begin{array}{l}33.8 \\
(30.9 \\
36.6)\end{array}$ & $\begin{array}{l}32.2(30.2 ; \\
34.6)\end{array}$ & $\begin{array}{l}28.7(27.6 \\
32.6) \star \star \star\end{array}$ \\
\hline WC (cm) & $109.0(9.1)$ & $\begin{array}{l}103.8 \\
(7.4)\end{array}$ & $\underset{* \star \star}{112.9}(8.3)$ & $\begin{array}{l}109.2 \\
(8.7)\end{array}$ & $108.7(9.6)$ & $99.9(9.5)$ \\
\hline DXA VAT (kg) & $\begin{array}{l}2.3(1.6 \\
3.1)\end{array}$ & $\begin{array}{l}2.2(1.5 \\
3.1)\end{array}$ & $\begin{array}{l}2.3(1.7 \\
3.1)\end{array}$ & $\begin{array}{l}2.3(1.7 \\
3.2)\end{array}$ & $\begin{array}{l}2.3(1.6 \\
3.0)\end{array}$ & 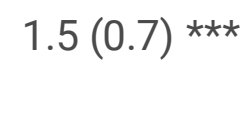 \\
\hline \multicolumn{7}{|l|}{$\begin{array}{l}\text { Biochemical } \\
\text { parameters }\end{array}$} \\
\hline TG (mg/dL) & $\begin{array}{l}\text { 121.0 (80.0; } \\
\text { 155.0) }\end{array}$ & $\begin{array}{l}92.0 \\
(72.0 \\
133.0)\end{array}$ & $\begin{array}{l}127.0 \\
(96.0 ; \\
170.0) \text { ** }\end{array}$ & $\begin{array}{l}126.0 \\
(80.0 ; \\
167.0)\end{array}$ & $\begin{array}{l}113.0 \\
(72.0 ; \\
148.0)\end{array}$ & $\begin{array}{l}83.0(56.0 \\
114.0) \star \star \star\end{array}$ \\
\hline $\begin{array}{l}\text { Glucose } \\
\text { (mg/dL) }\end{array}$ & $\begin{array}{l}101.5(91 ; \\
108)\end{array}$ & $\begin{array}{l}97.0 \\
(92.0 \\
105.0)\end{array}$ & $\begin{array}{l}103.0 \\
(91.0 ; \\
115.0)\end{array}$ & $\begin{array}{l}97.0 \\
(91.0 \\
104.0)\end{array}$ & $\begin{array}{l}104.0 \\
(95.0 ; \\
112.0) \text { * }\end{array}$ & $\begin{array}{l}92.0(87.0 \\
98.0) \star \star \star\end{array}$ \\
\hline Insulin (U/mL) & $16.6(7.9)$ & $15.3(7.8)$ & $17.7(7.9)$ & $15.4(7.1)$ & $17.9(8.5)$ & $\begin{array}{l}8.6(6.5 \\
13.5) * \star \star\end{array}$ \\
\hline HOMA-IR & $\begin{array}{l}4.1(2.8 \\
5.7)\end{array}$ & $\begin{array}{l}3.5(2.2 \\
5.1)\end{array}$ & $\begin{array}{l}4.4(3.1 \\
6.0) *\end{array}$ & $\begin{array}{l}3.9(2.6 \\
5.1)\end{array}$ & $\begin{array}{l}4.4(2.9 ; \\
6.5)\end{array}$ & $\begin{array}{l}1.9(1.4 \\
3.3) \star \star \star\end{array}$ \\
\hline TyG index & $8.6(0.5)$ & $8.4(0.4)$ & $\underset{\star \star \star \star}{8.8}(0.4)$ & $8.6(0.4)$ & $8.6(0.5)$ & $\begin{array}{l}8.2(7.8 \\
8.6) \star \star \star\end{array}$ \\
\hline
\end{tabular}

Variables re shown as mean (SD) or as median (IQR) according to its distribution. Categorical variables are presented as absolute (n). Paired t test and Wilcoxon-matched-pairs signed ranks were carried out to compare baseline and 6-months participants characteristics. Independent samples ttests and Wilcoxon-Mann-Whitney were carried out to compare changes between sex and age groups. Age was categorized according to the median. ${ }^{\star} p<0.05 ; * \star p<0.01$; ${ }^{* \star \star} p<0.001$. ${ }^{*}$ Comparison within dietary groups (baseline and after 6 months). BMI, Body Mass Index; DXA, Dual-Energy x-ray Absorptiometry; \%E, Percentage of Energy; FGF21, Fibroblast growth factor 21; FLI, Fatty Liver Index; HDL-c, High Density Lipoprotein-cholesterol; HOMA-IR, Homeostasis Model Assessment Insulin Resistance; MRI, Magnetic Resonance Imaging; OWL $\circledast$; OWLiver $\circledast$-test; PA, Physical activity; TG, Triglycerides; TyG index, Triglycerides and Glucose Index; VAT, Visceral Adipose Tissue; WC, Waist Circumference. 


\begin{tabular}{|c|c|c|c|c|c|c|}
\hline & Baseline & & & & & \\
\hline $\begin{array}{l}\text { Adiponectin } \\
(\mu \mathrm{g} / \mathrm{mL})\end{array}$ & $\begin{array}{l}6.3(5.0 \\
8.3)\end{array}$ & $\begin{array}{l}6.6(5.2 \\
8.3)\end{array}$ & $\begin{array}{l}5.9(5.0 \\
7.5)\end{array}$ & $\begin{array}{l}32.1 \\
(17.6 ; \\
46.09)\end{array}$ & $\begin{array}{l}26.4(15.9 \\
39.3) *\end{array}$ & $\begin{array}{l}8.0(6.1 \\
9.9)^{\star \star \star}\end{array}$ \\
\hline $\begin{array}{l}\text { Leptin } \\
\text { (ng/mL) }\end{array}$ & $\begin{array}{l}29.8(17.6 \\
44.8)\end{array}$ & $\begin{array}{l}46.0 \\
(37.7 \\
69.3)\end{array}$ & $\begin{array}{l}20.1(14.0 \\
26.4) \star \star \star \star\end{array}$ & $\begin{array}{l}5.8(4.5 \\
7.9)\end{array}$ & $\begin{array}{l}6.6(5.7 \\
9.7)\end{array}$ & $\begin{array}{l}16.7(7.5 \\
33.0) \star \star \star \star\end{array}$ \\
\hline $\begin{array}{l}\text { FGF21 } \\
(\mathrm{pg} / \mathrm{mL})\end{array}$ & $\begin{array}{l}211.5 \\
(108.0 ; \\
352.0)\end{array}$ & $\begin{array}{l}190.0 \\
(89.1 ; \\
387.0)\end{array}$ & $\begin{array}{l}215.0 \\
(124.0 ; \\
328.0)\end{array}$ & $\begin{array}{l}182.0 \\
(87.7 \\
302.0)\end{array}$ & $\begin{array}{l}244.0 \\
(130.0 ; \\
416.0)\end{array}$ & $\begin{array}{l}187.5 \\
(111.0 ; \\
355.0)\end{array}$ \\
\hline \multicolumn{7}{|l|}{ Liver injury } \\
\hline FLI & $\begin{array}{l}83.1(73.7 ; \\
92.3)\end{array}$ & $\begin{array}{l}76.0 \\
(60.7 \\
83.0)\end{array}$ & $\begin{array}{l}89.6(79.8 \\
94.1) \star \star \star\end{array}$ & $\begin{array}{l}84.4 \\
(74.2 \\
93.3)\end{array}$ & $\begin{array}{l}79.8(70.5 ; \\
91.7)\end{array}$ & $\begin{array}{l}51.11 \\
(23.6) * \star \star\end{array}$ \\
\hline $\begin{array}{l}\text { MRI Liver fat } \\
\text { - Dixon (\%) }\end{array}$ & $\begin{array}{l}5.6(3.2 ; \\
9.6)\end{array}$ & $\begin{array}{l}4.5(2.9 ; \\
8.7)\end{array}$ & $\begin{array}{l}6.5(4.3 \\
10.1) *\end{array}$ & $\begin{array}{l}5.9(3.5 \\
12.4)\end{array}$ & $\begin{array}{l}5.0(3.0 ; \\
8.9)\end{array}$ & $\begin{array}{l}2.0(1.3 \\
3.8) \star * \star\end{array}$ \\
\hline \multicolumn{7}{|c|}{$\begin{array}{l}\text { Lipidomic (OWLiver@-test) n } \\
(\%)\end{array}$} \\
\hline No NAFLD & $17(20.0)$ & 7 (18.9) & $10(20.8)$ & 7 (16.2) & $10(23.8)$ & $23(32.8)$ \\
\hline $\begin{array}{l}\text { Hepatic } \\
\text { Steatosis }\end{array}$ & $20(23.5)$ & $10(27.0)$ & $10(20.8)$ & 7 (16.2) & $13(30.9)$ & $21(30.0)$ * \\
\hline $\mathrm{NASH}$ & $48(56.4)$ & $20(54.0)$ & 28 (58.3) & $29(67.4)$ & $19(45.2)$ & $26(37.1)$ \\
\hline \multicolumn{7}{|l|}{$\begin{array}{l}\text { Dietary intake } \\
\text { per day }\end{array}$} \\
\hline $\begin{array}{l}\text { Total energy } \\
\text { (kcal/day) }\end{array}$ & $\begin{array}{l}2550 \text { (1958; } \\
2925)\end{array}$ & $\begin{array}{l}2548 \\
\text { (2031; } \\
3133)\end{array}$ & $\begin{array}{l}2554 \\
(1897 \\
2902)\end{array}$ & $\begin{array}{l}2551 \\
(2042 ; \\
3066)\end{array}$ & $\begin{array}{l}2464 \\
(1833 \\
2864)\end{array}$ & $2004(576)$ \\
\hline $\begin{array}{l}\text { Carbohydrates } \\
(\% \mathrm{E})\end{array}$ & $\begin{array}{l}42.8(37.6 \\
47.8)\end{array}$ & $\begin{array}{l}43.0 \\
(35.9 \\
48.4)\end{array}$ & $\begin{array}{l}42.5(39.2 ; \\
47.5)\end{array}$ & $\begin{array}{l}40.8 \\
(36.2 \\
46.8)\end{array}$ & $\begin{array}{l}43.0(39.9 ; \\
48.0)\end{array}$ & $42.3(7.7)$ \\
\hline
\end{tabular}

Variables re shown as mean (SD) or as median (IQR) according to its distribution. Categorical variables are presented as absolute (n). Paired t test and Wilcoxon-matched-pairs signed ranks were carried out to compare baseline and 6-months participants characteristics. Independent samples ttests and Wilcoxon-Mann-Whitney were carried out to compare changes between sex and age groups. Age was categorized according to the median. ${ }^{\star} p<0.05 ; * \star p<0.01$; ${ }^{* \star \star} p<0.001$. ${ }^{*}$ Comparison within dietary groups (baseline and after 6 months). BMI, Body Mass Index; DXA, Dual-Energy x-ray Absorptiometry; \%E, Percentage of Energy; FGF21, Fibroblast growth factor 21; FLI, Fatty Liver Index; HDL-c, High Density Lipoprotein-cholesterol; HOMA-IR, Homeostasis Model Assessment Insulin Resistance; MRI, Magnetic Resonance Imaging; OWL®; OWLiver® -test; PA, Physical activity; TG, Triglycerides; TyG index, Triglycerides and Glucose Index; VAT, Visceral Adipose Tissue; WC, Waist Circumference. 


\begin{tabular}{|c|c|c|c|c|c|c|}
\hline \multicolumn{7}{|c|}{ Baseline } \\
\hline Proteins (\%E) & $\begin{array}{l}16.8(15.1 \\
19.1)\end{array}$ & $\begin{array}{l}16.9 \\
(15.2 \\
20.9)\end{array}$ & $\begin{array}{l}\text { 16.7 (15.1; } \\
19.0)\end{array}$ & $\begin{array}{l}16.7 \\
(15.3 \\
18.8)\end{array}$ & $\begin{array}{l}16.9(14.6 ; \\
19.3)\end{array}$ & $\begin{array}{l}19.4(17.1 \\
22.8) \star \star \star \star\end{array}$ \\
\hline Fats (\%E) & $37.4(6.8)$ & $38.1(7.5)$ & 36.8 (6.2) & $38.3(6.8)$ & $36.5(6.7)$ & $35.4(7.8)$ \\
\hline \multicolumn{7}{|l|}{$\begin{array}{l}\text { Lifestyle } \\
\text { factors }\end{array}$} \\
\hline MedDiet Score & $5.9(1.9)$ & $5.9(2.3)$ & $5.9(1.6)$ & $5.4(1.7)$ & $6.4(2.0)$ * & $\begin{array}{l}12.0(10.0 \\
14.0) * \star * \star\end{array}$ \\
\hline $\begin{array}{l}\text { PA (METs- } \\
\text { min/week) }\end{array}$ & $\begin{array}{l}2240 \text { (1665; } \\
4307)\end{array}$ & $\begin{array}{l}2240 \\
(1710 ; \\
4307)\end{array}$ & $\begin{array}{l}2280 \\
(1100 ; \\
4365)\end{array}$ & $\begin{array}{l}2322 \\
(1705 ; \\
4365)\end{array}$ & $\begin{array}{l}2216 \\
(1392 \\
4200)\end{array}$ & $\begin{array}{l}3720(2442 ; \\
5115) \text { * }\end{array}$ \\
\hline \multicolumn{7}{|c|}{ 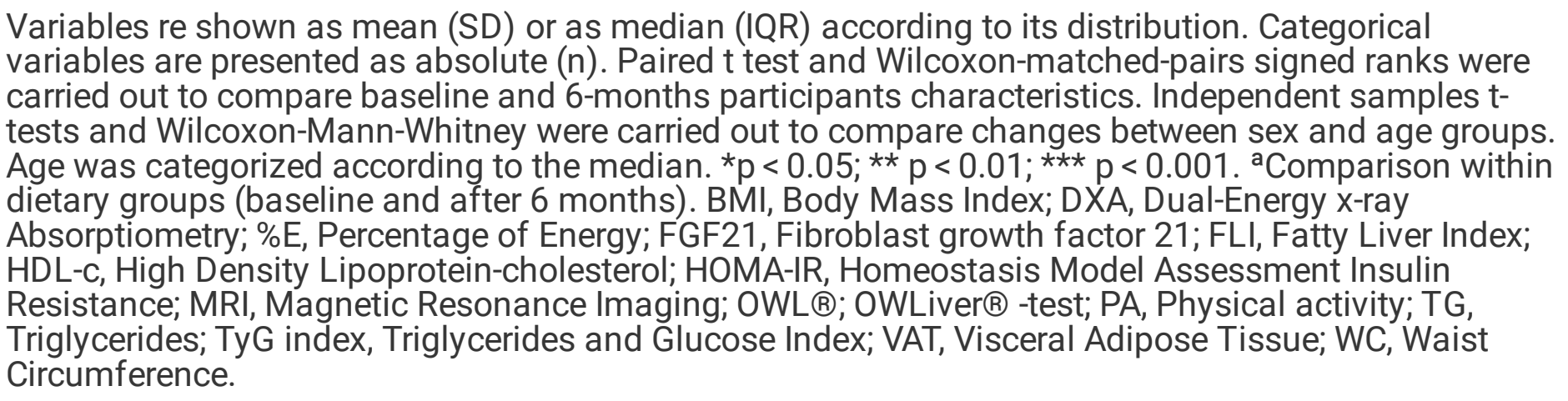 } \\
\hline
\end{tabular}

To study the genetic risk association with NAFLD, of a total of 95 genotyped related-obesity SNPs, 38 obesity-related genetic variants were chosen because they were statistically or marginally associated with the amelioration of the hepatic health measured by non-invasive NAFLD diagnostic methods (FLI, MRI and OWLiver ${ }^{\circledR}$-test). Of those, 1 SNP was common among all methods: rs2959272 (PPARG). On the other hand, 30 SNPs were exclusively related to a specific method-17 for FLI rs1801133 (MTHFR), rs 1055144 (NFE2L3), rs17817449 (FTO), rs8050136 (FTO), rs3751812 (FTO), rs9939609 (FTO), rs2075577 (UCP3), rs324420 (FAAH), rs1121980 (FTO), rs2419621 (ACSL5), rs1558902 (FTO), rs3123554 (CNR2/FUCA1), rs6567160 (MC4R), rs660339 (UCP2), rs2605100 (LYPLAL 1), rs1800629 (TNFAPROMOTOR), rs4994 (ADRB3); 3 for MRI: rs6861681 (CPEB4), rs1440581 (PPM1K), rs1799883 (FABP2); and 10 for OW-Liver test: rs1175544 (PPARG), rs1797912 (PPARG), rs1386835 (PPARG), rs709158 (PPARG), rs 1175540 (PPARG), rs1801260 (CLOCK), rs12502572 (UCP1), rs8179183 (LEPR), rs894160 (PLIN1), rs4731426 $(L E P)$. In our population we used the SNP associated with each non-invasive method for calculating the GRS (Figure S1).

The GRS, calculated as the number of risk alleles carried by each subject, was normally distributed. The sample was stratified, by the median, into a "low genetic risk group," those with a $\mathrm{GRS}_{\mathrm{FLI}} \leq 9, \mathrm{GRS}_{\mathrm{OWL}} \leq$ 10 and $\mathrm{GRS}_{\mathrm{MRI}} \leq 4$ risk alleles, and into a "high genetic risk group," those with a $\mathrm{GRS}_{\mathrm{FLI}}>9, \mathrm{GRS}_{\mathrm{OWL}}>10$ and $\mathrm{GRS}_{\mathrm{MRI}}>4$ risk alleles. The results for the effect of each GRS on the change of different outcomes 
after the nutritional treatment are shown (Table 2). All groups exhibited a significant body weight loss, which was higher when the genetic risk was lower. Moreover, body composition variables including weight, BMI and waist circumference showed statistical differences when comparing $\mathrm{GRS}_{\mathrm{FLI}}$ and $\mathrm{GRS}_{\mathrm{MRI}}$ medians. Furthermore, general improvements in biochemical parameters were found. However, the amelioration was only statistically significant in TG, TyG and leptin concentrations and TG and FGF-21 concentrations, when comparing $\mathrm{GRS}_{\mathrm{FLI}}$ and $\mathrm{GRS}_{\mathrm{MRI}}$ medians respectively. On the other hand, no significant changes were found for dietary intake and lifestyle factors. 
Table 2

Change in body composition, biochemical, dietary and lifestyle factors according to different Genetic Risk Scores

\begin{tabular}{|c|c|c|c|c|c|c|}
\hline & \multicolumn{2}{|l|}{$\mathrm{GRS}_{\mathrm{FL}}$} & \multicolumn{2}{|l|}{ GRS $_{\mathrm{OWL}}$} & \multicolumn{2}{|l|}{$\mathrm{GRS}_{\mathrm{MRI}}$} \\
\hline & $<9$ & $\geq 9$ & $<10$ & $\geq 10$ & $<4$ & $\geq 4$ \\
\hline$n$ & 30 & 40 & 29 & 41 & 31 & 39 \\
\hline \multicolumn{7}{|l|}{$\begin{array}{l}\text { Body } \\
\text { composition }\end{array}$} \\
\hline Mean & $6.0(1.6)$ & $9.6(3.9)$ & $7.0(1.5)$ & $11.7(1.4)$ & $2.0(0.8)$ & $4.9(1.0)$ \\
\hline$\Delta$ Weight $(\mathrm{kg})$ & $\begin{array}{l}-10.6(-15.8 ; \\
-6.9)\end{array}$ & $\begin{array}{l}-8.4(-10.7 \\
-5.0) \text { * }\end{array}$ & $\begin{array}{l}-9.5 \\
(-12.4 \\
-6.4)\end{array}$ & $\begin{array}{l}-8.6(-12 \\
-6.4)\end{array}$ & $\begin{array}{l}-9.6(-16.9 ; \\
-8.2)\end{array}$ & $\begin{array}{l}-7.6(-11.2 \\
-4.9) *\end{array}$ \\
\hline$\Delta \mathrm{BMI}\left(\mathrm{kg} / \mathrm{m}^{2}\right)$ & $\begin{array}{l}-3.6(-5.1 \\
-2.4)\end{array}$ & $\begin{array}{l}-2.9(-3.7 \\
-1.6)^{*}\end{array}$ & $\begin{array}{l}-3.3(-4.7 ; \\
-2.2)\end{array}$ & $\begin{array}{l}-3.2(-4.1 \\
-2.2)\end{array}$ & $\begin{array}{l}-3.5(-5.6 \\
-2.9)\end{array}$ & $\begin{array}{l}-2.8(-3.8 \\
-1.6)^{\star}\end{array}$ \\
\hline$\Delta \mathrm{WC}(\mathrm{cm})$ & $-11.7(6.5)$ & $\begin{array}{l}-7.6(5.6) \\
\star \star\end{array}$ & $-9.1(6.4)$ & $-9.5(6.3)$ & $-11.0(5.2)$ & $-8.0(6.8)$ * \\
\hline$\triangle \mathrm{DXA}$ VAT $(\mathrm{kg})$ & $\begin{array}{l}-0.8(-1.2 \\
-0.4)\end{array}$ & $\begin{array}{l}-0.9(-1.5 \\
-0.4)\end{array}$ & $\begin{array}{l}-1.0(-1.5 \\
-0.4)\end{array}$ & $\begin{array}{l}-0.8(-1.5 \\
-3.2)\end{array}$ & $\begin{array}{l}-1.0(-1.5 \\
-0.6)\end{array}$ & $\begin{array}{l}-0.7(-1.67 ; \\
-0.2)\end{array}$ \\
\hline \multicolumn{7}{|l|}{$\begin{array}{l}\text { Biochemical } \\
\text { parameters }\end{array}$} \\
\hline$\Delta \mathrm{TG}(\mathrm{mg} / \mathrm{dL})$ & $\begin{array}{l}-42.0 \\
(-100.0 \\
-18.0)\end{array}$ & $\begin{array}{l}-15.0 \\
(-56.0 ; 4.0) \\
\star\end{array}$ & $\begin{array}{l}-32.0 \\
(-68 ; 0)\end{array}$ & $\begin{array}{l}-22.5 \\
(-58.5 \\
-0.5)\end{array}$ & $\begin{array}{l}-46.0 \\
(-102.0 \\
-5.0)\end{array}$ & $\begin{array}{l}-18.0 \\
(-45.0 ; 1.0) \\
\star\end{array}$ \\
\hline $\begin{array}{l}\Delta \text { Glucose } \\
\text { (mg/dL) }\end{array}$ & $-8.6(11.0)$ & $\begin{array}{l}-10.2 \\
(12.4)\end{array}$ & $\begin{array}{l}-9.8 \\
(13.6)\end{array}$ & $-9.3(10.4)$ & $-9.2(11.0)$ & $-9.8(12.5)$ \\
\hline$\Delta$ Insulin $(\mathrm{U} / \mathrm{mL})$ & $-7.4(7.1)$ & $-4.9(8.0)$ & $-6.2(8.7)$ & $-5.9(7.0)$ & $-6.7(7.4)$ & $-5.4(8.0)$ \\
\hline$\triangle \mathrm{HOMA}-\mathrm{IR}$ & $\begin{array}{l}-2.0(-3.2 ; \\
-0.2)\end{array}$ & $\begin{array}{l}-1.6(-3.3 ; \\
-0.2)\end{array}$ & $\begin{array}{l}-1.9(-3.2 ; \\
-0.2)\end{array}$ & $\begin{array}{l}-1.8(-3.2 ; \\
-0.6)\end{array}$ & $\begin{array}{l}-2.3(-3.2 \\
-1.0)\end{array}$ & $\begin{array}{l}-1.5(-3.2 ; \\
-0.1)\end{array}$ \\
\hline$\Delta$ TyG index & $-0.6(0.4)$ & $\frac{-0.2(0.4)}{\star \star}$ & $-0.4(0.3)$ & $-0.4(0.5)$ & $-0.5(0.5)$ & $-0.3(0.3)$ \\
\hline
\end{tabular}

Variables are shown as mean (SD) or as median (IQR) according to its distribution. Categorical variables are presented as absolute (n). Independent samples t-tests and Wilcoxon-Mann-Whitney were carried out to compare variables changes according to the median of $\mathrm{GRS}_{\mathrm{FLI}}<9$ and $\mathrm{GRS}_{\mathrm{FLI}} \geq 9$, $\mathrm{GRS}_{\mathrm{MRI}}<4$ and $\mathrm{GRS}_{\mathrm{MRI}} \geq 4$ and $\mathrm{GRS}_{\mathrm{OWL}}<10$ and $\mathrm{GRS}_{\mathrm{OWL}} \geq 10 .{ }^{\star} \mathrm{p}<0.05 ; * \star p<0.01 ; * \star \star p<0.001$. BMI, Body Mass Index; DXA, Dual-Energy x-ray Absorptiometry; \%E, Percentage of Energy; FGF21, Fibroblast growth factor 21; FLI, Fatty Liver Index; HDL-c, High Density Lipoprotein-Cholesterol; HOMAIR, Homeostasis Model Assessment Insulin Resistance; MRI, Magnetic Resonance Imaging; OWL $\circledast$; OWLiver ${ }^{\circledR}$-test; PA, Physical activity; TG, Triglycerides; TyG index, Triglycerides and Glucose Index; VAT, Visceral Adipose Tissue; WC, Waist Circumference. 


\begin{tabular}{|c|c|c|c|c|c|c|}
\hline & $\mathrm{GRS}_{\mathrm{FL}}$ & & $\mathrm{GRS}_{\mathrm{OWL}}$ & & $\mathrm{GRS}_{\mathrm{MRI}}$ & \\
\hline $\begin{array}{l}\Delta \text { Adiponectin } \\
(\mu \mathrm{g} / \mathrm{mL})\end{array}$ & $\begin{array}{l}1.5(0.1 ; \\
4.7)\end{array}$ & $\begin{array}{l}1.2(-0.9 \\
3.2)\end{array}$ & $\begin{array}{l}-0.1(-0.6 \\
2.2)\end{array}$ & $\begin{array}{l}1.8(0.1 ; \\
3.4)\end{array}$ & $\begin{array}{l}1.0(-0.6 \\
3.4)\end{array}$ & $\begin{array}{l}1.3(-0.2 \\
4.1)\end{array}$ \\
\hline $\begin{array}{l}\Delta \text { Leptin } \\
(\mathrm{ng} / \mathrm{mL})\end{array}$ & $\begin{array}{l}-11.1(-21.6 ; \\
-7.2)\end{array}$ & $\begin{array}{l}-7.5(-14.5 \\
-2.9) *\end{array}$ & $\begin{array}{l}-9.5 \\
(-15.8 \\
-7.0)\end{array}$ & $\begin{array}{l}-7.5(-20.0 \\
-3.0)\end{array}$ & $\begin{array}{l}-9.1(-15.8 ; \\
-6.7)\end{array}$ & $\begin{array}{l}-9.1(-20.0 ; \\
-3.0)\end{array}$ \\
\hline $\begin{array}{l}\Delta \mathrm{FGF} 21 \\
(\mathrm{pg} / \mathrm{mL})\end{array}$ & $\begin{array}{l}-9.1(-123.0 \\
80.0)\end{array}$ & $\begin{array}{l}-40.5 \\
(-146.5 \\
95.5)\end{array}$ & $\begin{array}{l}-41.7 \\
(-132 ; 50)\end{array}$ & $\begin{array}{l}-0.8(-123 \\
88)\end{array}$ & $\begin{array}{l}-55.4 \\
(-217 ; 45)\end{array}$ & $\begin{array}{l}12.0 \\
(-64.2 ; 97) \\
*\end{array}$ \\
\hline$\Delta \mathrm{FLI}(\%)$ & $-54.5(19.7)$ & $\begin{array}{l}-22.6 \\
(17.9) \star \star \star\end{array}$ & $\begin{array}{l}-33.7 \\
(21.8)\end{array}$ & $\begin{array}{l}-38.4 \\
(26.2)\end{array}$ & $\begin{array}{l}-41.1 \\
(25.3)\end{array}$ & $\begin{array}{l}-32.9 \\
(23.5)\end{array}$ \\
\hline $\begin{array}{l}\Delta M R I \text { Liver fat - } \\
\text { Dixon (\%) }\end{array}$ & $\begin{array}{l}-2.7(-6.8 \\
-0.7)\end{array}$ & $\begin{array}{l}-2.7(-6.8 \\
-1.2)\end{array}$ & $\begin{array}{l}-4.3(-8 \\
-0.8)\end{array}$ & $\begin{array}{l}-3.4(-6.8 \\
-1.2)\end{array}$ & $\begin{array}{l}-4.5(-7.8 \\
-2.5)\end{array}$ & $\begin{array}{l}-1.6(-4.2 ; \\
-0.2)^{\star \star \star \star}\end{array}$ \\
\hline \multicolumn{7}{|c|}{$\begin{array}{l}\Delta \text { Lipidomic (OWLiver@-test) n } \\
(\%)\end{array}$} \\
\hline $\begin{array}{l}\mathrm{OWL}^{\circledR} \\
\text { maintenance }\end{array}$ & $19(63.3)$ & 28 (71.7) & $13(44.8)$ & $34(85.0)$ & $17(56.6)$ & $30(76.9)$ \\
\hline OWL ${ }^{\circledR}$ reduction & $11(36.6)$ & $11(28.2)$ & $16(55.1)$ & $6(15.0)$ & 13 (43.3) & $9(23.0)$ \\
\hline \multicolumn{7}{|l|}{$\begin{array}{l}\text { Dietary intake } \\
\text { per day }\end{array}$} \\
\hline $\begin{array}{l}\Delta \text { Total energy } \\
(\mathrm{kcal})\end{array}$ & $\begin{array}{l}-882 \\
(-1261 ;-88)\end{array}$ & $\begin{array}{l}-523 \\
(-1099 \\
-101)\end{array}$ & $\begin{array}{l}-589 \\
(-987 \\
-132)\end{array}$ & $\begin{array}{l}-603 \\
(-1175 \\
44)\end{array}$ & $\begin{array}{l}-881 \\
(-1257 \\
-308)\end{array}$ & $\begin{array}{l}-479 \\
(-1009 ; 66)\end{array}$ \\
\hline $\begin{array}{l}\Delta \text { Carbohydrates } \\
(\%)\end{array}$ & $-1.3(10.0)$ & $-0.7(8.7)$ & $-1.9(8.2)$ & $-0.3(9.9)$ & $-2.0(9.4)$ & $-0.3(9.1)$ \\
\hline$\Delta$ Proteins (\%) & $3.6(4.3)$ & $1.9(5.7)$ & $2.5(3.5)$ & $2.7(6.1)$ & $2.9(6.0)$ & $2.4(4.6)$ \\
\hline$\Delta$ Fats $(\%)$ & $\begin{array}{l}-0.7(-6.2 \\
4.5)\end{array}$ & $\begin{array}{l}-2.1(-9.1 \\
5.1)\end{array}$ & $\begin{array}{l}-0.2(-5.2 \\
5.2)\end{array}$ & $\begin{array}{l}-1.8(-10.1 ; \\
4.8)\end{array}$ & $\begin{array}{l}-2.6(-8.3 \\
4.1)\end{array}$ & $\begin{array}{l}-1.4(-9.8 \\
5.8)\end{array}$ \\
\hline \multicolumn{7}{|l|}{ Lifestyle factors } \\
\hline$\Delta$ MedDiet Score & $6.3(3.1)$ & $5.7(3.4)$ & 5.7 (3.2) & $6.1(3.4)$ & $6.5(3.3)$ & $5.5(3.3)$ \\
\hline
\end{tabular}

Variables are shown as mean (SD) or as median (IQR) according to its distribution. Categorical variables are presented as absolute (n). Independent samples t-tests and Wilcoxon-Mann-Whitney were carried out to compare variables changes according to the median of $\mathrm{GRS}_{\mathrm{FL}}<9$ and $\mathrm{GRS}_{\mathrm{FL}} \geq 9$, $\mathrm{GRS}_{\mathrm{MRI}}<4$ and $\mathrm{GRS}_{\mathrm{MRI}} \geq 4$ and $\mathrm{GRS}_{\mathrm{OWL}}<10$ and $\mathrm{GRS}_{\mathrm{OWL}} \geq 10 .{ }^{*} \mathrm{p}<0.05 ;{ }^{* \star} \mathrm{p}<0.01 ;{ }^{* \star *} \mathrm{p}<0.001$. BMI, Body Mass Index; DXA, Dual-Energy x-ray Absorptiometry; \%E, Percentage of Energy; FGF21, Fibroblast growth factor 21; FLI, Fatty Liver Index; HDL-C, High Density Lipoprotein-Cholesterol; HOMAIR, Homeostasis Model Assessment Insulin Resistance; MRI, Magnetic Resonance Imaging; OWL $\circledast$; OWLiver ${ }^{\circledR}$-test; PA, Physical activity; TG, Triglycerides; TyG index, Triglycerides and Glucose Index; VAT, Visceral Adipose Tissue; WC, Waist Circumference. 


\begin{tabular}{|c|c|c|c|c|c|c|}
\hline \multicolumn{3}{|c|}{$\mathrm{GRS}_{\mathrm{FLI}}$} & \multicolumn{2}{|l|}{$\mathrm{GRS}_{\mathrm{OWL}}$} & \multicolumn{2}{|l|}{$\mathrm{GRS}_{\mathrm{MRI}}$} \\
\hline $\begin{array}{l}\Delta \mathrm{PA} \text { (METs- } \\
\mathrm{min} / \text { week) }\end{array}$ & $\begin{array}{l}758(-217 \\
2405)\end{array}$ & $\begin{array}{l}1215(-120 ; \\
2798)\end{array}$ & $\begin{array}{l}896(73 \\
2798)\end{array}$ & $\begin{array}{l}1111 \\
(-753 \\
2405)\end{array}$ & $\begin{array}{l}984(-65 \\
2357)\end{array}$ & $\begin{array}{l}1046 \\
(-557 \\
2817)\end{array}$ \\
\hline \multicolumn{7}{|c|}{ 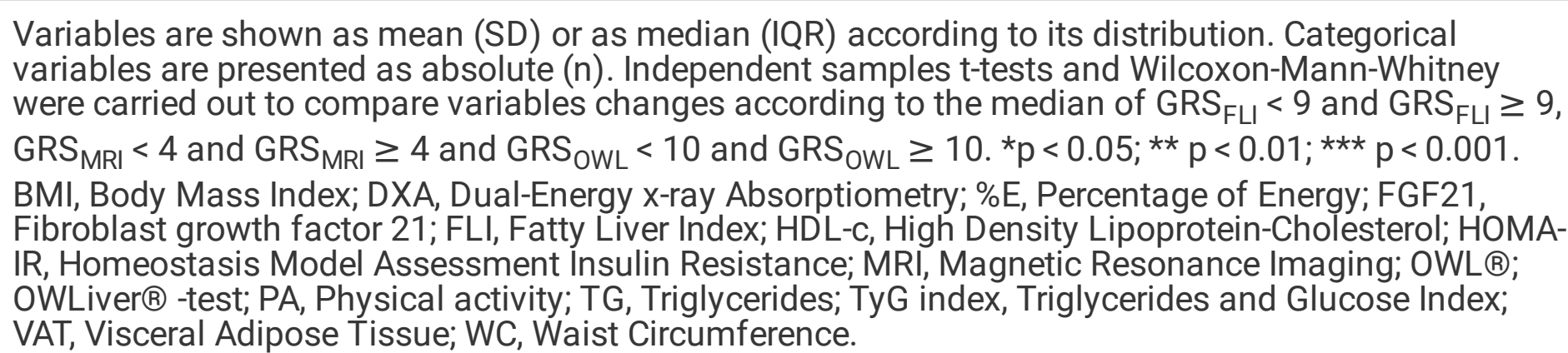 } \\
\hline
\end{tabular}

In order to evaluate the improvement of hepatic health depending on genetic and non-genetic risk factors, linear regression models were constructed (Table 3). These models were adjusted for sex, age, baseline protein intake, baseline FGF-21 and insulin concentration and the change in MedDiet Score. All the GRS included in the models showed an important association with the improvement on hepatic health. Moreover, a higher decrease in FLI was significantly associated with baseline insulin and protein and with the change in MedDietScore, showing an improvement in hepatic functionality. Besides, a high intake of protein at baseline seemed to be also important in the improvement of lipid metabolism, assessed by OWLiver ${ }^{\circledR}$-test. Parallelly, the regression model stablished for the change on liver fat content (MRI) showed a significant interaction between the $\mathrm{GRS}_{\mathrm{MRI}}$ and protein intake at baseline ( $p$-value: 0.001). However, no statistically significant interactions between $\mathrm{GRS}_{\mathrm{FL}}$ and $\mathrm{GRS}_{\mathrm{OWL}}$ were found. Overall, the change of FLI, OWLiver $\AA$-test and MRI variabilities were explained in approximately $52 \%\left(\operatorname{adj} . R^{2}=0.53\right)$, $16 \%\left(\operatorname{adj} . R^{2}=0.16\right)$ and $34 \%\left(\right.$ adj. $\left.R^{2}=0.34\right)$, respectively. 
Table 3

Linear regression analyses of changes in Fatty Liver Index, OWLiver®-test and liver fat content (MRI)

$\beta$

\% Change in Fatty Liver Index

(FLI)

Model 1

Model 2

Model 3

Model 4

\begin{tabular}{l}
\hline Change in OWLiver ${ }^{\circledR}$-test \\
\hline Model 5 \\
\hline Model 6 \\
\hline $\begin{array}{l}\text { Change in liver fat content } \\
\text { (MRI) }\end{array}$
\end{tabular}

Model 7

Model 8

$$
\mathrm{GRS}_{\mathrm{MRI}}
$$

1.13

$<$

0.001

1.28

$<$

$\mathrm{GRS}_{\mathrm{MRI}}$

0.001

0.24

0.741
Adjusted

$\mathrm{R}^{2}$

PModel 


\begin{tabular}{|c|c|c|c|c|c|}
\hline & & $\beta$ & $\begin{array}{l}\mathrm{p}- \\
\text { value }\end{array}$ & $\begin{array}{l}\text { Adjusted } \\
\mathrm{R}^{2}\end{array}$ & $\begin{array}{l}\text { p- } \\
\text { Model }\end{array}$ \\
\hline \multirow[t]{3}{*}{ Model 9} & $\mathrm{GRS}_{\mathrm{MRI}}$ & 1.17 & $\begin{array}{l}< \\
0.001\end{array}$ & 0.28 & $\begin{array}{l}< \\
0.001\end{array}$ \\
\hline & Baseline protein & 0.09 & 0.448 & & \\
\hline & Baseline FGF21 & -0.004 & 0.051 & & \\
\hline \multirow[t]{2}{*}{ Model 10} & $\mathrm{GRS}_{\mathrm{MRI}} \#$ baselineprotein & 0.180 & 0.017 & 0.34 & $\begin{array}{l}< \\
0.001\end{array}$ \\
\hline & Baseline FGF21 & -0.004 & 0.040 & & \\
\hline \multicolumn{6}{|c|}{$\begin{array}{l}\text { All models were adjusted by age and sex. GRS } \mathrm{FLI} \text {, Genetic Risk Score for FLl; GRS } \mathrm{MRl} \text {, Genetic Risk } \\
\text { Score for Magnetic Resonance Imaging; GRS }{ }_{\mathrm{OWL}} \text { Genetic Risk Score for OWLiver®-test; MRI, } \\
\text { Magnetic Resonance Imaging. }\end{array}$} \\
\hline
\end{tabular}

In addition, Fig. 1A-C plots simple linear regression analyses of statistically significant predictors of FLI, OWLiver ${ }^{\circledR}$-test and liver fat content (MRI) decrease by diet. A lower change on FLI and OWLiver ${ }^{\circledR}$-test was associated with a higher baseline protein intake ( $p$-value: 0.009 and 0.022 , respectively). Moreover, this association became more important when the genetic risk was higher (Fig. 1A and B). Besides, Fig. 1C showed that a higher baseline protein was associated with a lower change of liver fat content (by MRI) becoming the effect more evident when the genetic risk was higher ( $p$ interaction: 0.017).

\section{Discussion}

NALFD has reached pandemic levels being recognized as an important health burden with an urgent need for early diagnosis [55]. Genetic predisposition for NAFLD has been reported $[17,19]$. With this in mind, the objective of this research was to assess the impact of the interaction between genetic and nongenetic factors concerning the improvement of the hepatic health using different diagnosis tools (FLI, MRI and OWLiver®-test) after a 6-month energy-restricted nutritional treatment for a more personalize management of this liver disease.

The high prevalence of NAFLD could be related to its strong link with obesity, which seems to play a role in both the initial simple steatosis and in its progression to NASH $[11,56]$. In this context, various genes and less frequent variants have been associated with the regulation of energy metabolism [17, 57]. Moreover, the increasing of knowledge of the genetic component of NAFLD has promoted the development of noninvasive diagnosis methods based on Genome Wide Associations Studies (GWAs) $[11,15,58-60]$, but few of them have examined the contribution of obesity-related variants linked to the evolution of this hepatic disease $[35,61]$. 
In order to better understand the contribution of genetics in the context of NAFLD three different GRS were constructed based on the improvement of hepatic health after an energy-restricted treatment measured by three non-invasive diagnostic methods (FLI, Magnetic Resonance Imaging and OWLiver ${ }^{\circledR}$ test). On the one hand, Fatty Liver Index has been highly correlated with measures of fatty liver disease showing an area under the curve of 0.84 , predicting most cases of NAFLD [62,63]. Moreover, a recent study reported that the FLI joint to the waist circumference-to-height ratio could be one of the most accurate algorithms for the noninvasive diagnosis of NAFLD in both lean and overweight/obese population $[64,65]$. On the other hand, MRI can be considered the gold standard for steatosis measurement being highly accurate and reproducible and superior in detecting and quantifying fat accumulation $[61,66]$. However, these two methods have limitations in detecting inflammation, ballooning and cellular injury, which are key components in NASH diagnosis [67]. Thus, in some cases, models base on "omics" sciences such as the OWLiver®-test, could be of interest adding knowledge about diverse factors influencing weight-loss variability among individuals. Due to the differences between methods in outcome measures, distinct genes and so pathways may be expected to be connected.

Therefore, a total of 38 polymorphisms were independently associated with differential responses to hepatic functionality (FLI), fat liver content (MRI) and lipid metabolism (OWLiver ${ }^{\circledR}$-test). It is important to emphasize that each non-invasive diagnostic method has its specific SNPs. Only the rs2959272 (PPARG) genetic variant was the common element on the three GRS. In this sense, an intervention study indicated that the PPARG genotype was associated with success in body weight reduction [68]. Indeed, two common elements were also observed between $\mathrm{GRS}_{\mathrm{FLI}}$ and $\mathrm{GRS}_{\mathrm{MRl}}$, SNPs were located in genes related to bile secretion ( $A B C B 11)$ and the regulation of energy balance and body weight (SH2B1). Meanwhile, SNPs in genes implicated in weight loss (SH2B1 and STK33) influenced both GRS OWL $_{\text {and }}$ GRS MRI. . Instead, 3 common elements mapped to genes involved in endocrine/enzymatic regulation of lipid metabolism affecting macronutrient (GNAS) food intake and energy expenditure (MC4R) and thermogenesis (UCP1) were observed in $\mathrm{GRS}_{\mathrm{FL}}$ and $\mathrm{GRS}_{\mathrm{OWL}}$, clarify the detailed path-way between body weight changes and the functions of the PPARG gene and SNPs

In this study, a greater change in most of the NAFLD-related variables was reported when the genetic risk was lower. According to these findings, it has been extensively debated the identification of the physiological pathways that control energy metabolism and body weight regulation $[29,69,70]$. A Genetic Investigation of ANtropometric Traits consortium (GIANT) metanalysis identified 97 loci for BMI where genes near these specific loci showed expression enrichment in the central nervous system, suggesting that BMI is mainly regulated by processes such as hypothalamic control of energy intake [71]. Similar results have been found in a recent study in a pediatric population, where the application of a GRS to established clinical risk factors significantly improved the discriminatory capability of the prediction of NAFLD risk [5]. Indeed, different genetic variants and interactions with environmental factors have been shown to modulate the differential individual responses to moderately high-protein and low-fat dietary interventions in a Caucasian population [53]. In this sense, genetic information could help to determine 
the most appropriate dietary intervention for the prevention and treatment of NAFLD, as well as the development of associated comorbidities [72,73]

Moreover, for the purpose of explaining the variability on the improvement in hepatic functionality (FLI), liver fat content (by MRI) and lipidomic (OWLiver ${ }^{\circledR}$-test), linear regression models were performed. The predictive accuracy of all models substantially improved when combining each of the previously mentioned SNPs in the multiple linear regression models, which is in line with previous studies[74]. In order to improve these results, each regression model was fitted by sex, age and NAFLD related variables such as inflammatory biomarkers or dietary compounds. Factors related with proinflammatory and profibrogenetic pathways such as leptin, adiponectin, or FGF-21, which appears to be elevated in patients with NAFLD, are therefore a promising target for the treatment $[75,76]$. Thus, $\mathrm{GRS}_{\mathrm{FL}}, \mathrm{GRS}_{\mathrm{MRI}}$ and GRS $\mathrm{OWL}$ were major predictor of the change in FLI, liver fat content (MRI) and OWLiver ${ }^{\circledR}$-test, respectively.

To the best of our knowledge, there are few studies showing the combined effects of GRS built from SNPs related weight and adiposity regulation in response to different energy-restricted diets $[48,77]$. Moreover, it has been reported that the genetic background is an important factor explaining metabolically health and unhealthy phenotypes related to obesity, in addition to lifestyle variables[52].

In this sense, dietary factors seem to be of key importance and have been associated with weight gain, obesity and NAFLD development $[78,79]$. Interestingly, in this research, higher baseline protein was associated with worst hepatic health improvement measured by FLI and OWLiver®-test. Furthermore, an interaction between the liver fat content assessed by MRI and baseline protein was found. In the same line were the results obtained from the Nurse's Health Study and the Health Professionals Follow-up Study where an increased intake of sugars-sweetened beverages was found to amplify the association of a 32-SNP genetic risk score with BMI [80]. These findings suggest that not only genetic and dietary factor should be considered but also the interaction of both of them [81-83]. Hence, a combined analysis over 16,000 children and adolescent showed the FTO rs9939609 variant that confers a predisposition to higher $\mathrm{BMI}$ is associated with higher total energy intake and that lower dietary protein intake attenuates the association [84].Among the macronutrient categories, protein is the main one that contributes to the satiety, contributing therefore to weight-loss $[85,86]$. However, the effect of the high protein diet in patients with NAFLD remain controversial. On the one hand, it has been suggested that the consumption of specific dietary amino acids might negatively impact on liver status and, to a lesser extent on glucose metabolism in subjects with overweight/obesity and NAFLD [87]. Moreover, high protein intake derived mainly from dairy products has been associated with higher risk to develop diabetes and also with NAFLD [88]. A recent study has also suggested that following a lower protein diet, particularly in genetically predisposed individuals, might be an effective approach for addressing cardiometabolic diseases among Southeast Asian women [89]. On the other hand, high protein diet has been reported to be a valid therapeutic approach to revert NAFLD, being of special importance the protein source and the functional status of the liver [90]. Besides, BCAA supplementation has been demonstrated to ameliorate liver fibrosis and suppress tumor growth in a rat model of HCC with liver cirrhosis, as well as alleviate hepatic steatosis and liver injury in NASH mice [91, 92]. 
As for drawbacks of this research: Firstly, liver biopsy results were not available to corroborate the precise diagnosis of patients [60]. Nonetheless, in this research we carried out a complete evaluation of the liver status by means of validated and widely used techniques as well as blood biomarkers and hepatic indexes, which are affordable and practical methods to use in public health settings. Second, the sample size and the enrollment of subjects are not very large. For this reason, these models should be further validated in different populations, to establish whether it indeed represents a reliable and accurate, "noninvasive alternative" to liver biopsy, as well as the role of new SNPs associated with excessive adiposity and accompanying metabolic alterations through a GRS approach needs to be explored. Thirdly, type I and type II errors cannot be completely ruled out, especially those related to the selection of SNPs to be introduced into the GRS. However, due to the use of less stringent $P$ value thresholds compared to association studies of single variants, genomic profile risk scoring analyses can tolerate, at balance, some of these biases, as previously reviewed [93]. Fourthly, dietary intake was evaluated using selfreported information of the participants, which may produce some bias on the evaluation of the results. Lastly the constructions of the GRS using specific obesity-related SNP it is also an important limitation. However, the inclusion of these SNP on the evaluation of the genetic influence on NAFLD could be also considered an important strength of this investigation, as well as the use of different multiple linear regression models to test the contribution of genetic, baseline protein and inflammatory factors on the management of NAFLD. Finally, the study is a randomized controlled trial where each volunteer has had an individual follow-up promoting the adoption of behavioral changes and a healthy lifestyle.

Overall, this experiment was designed as a proof of concept in order to evaluate if the genetic background linked to NAFLD-related factors may influence hepatic amelioration. In addition, examining new causes of disease and the underlying mechanism or alteration in specific pathways and clinical outcomes may be of interest.

\section{Conclusions}

Predicting the individual risk of NAFLD and determining the probability of disease progression is the basis for a precision diagnosis and treatment. These results demonstrate that three different genetic scores can be useful for the personalized management of NAFLD, whose treatment must rely on specific dietary recommendations guided by the measurement of specific genetic biomarkers.

\section{Abbreviations}

\section{AASLD}

American Association for the Study of Liver Diseases

AHA

American Heart Association

ALT

Alanine Aminotransferase

AST 
Aspartate Aminotransferase

BCAA

Branched-chain amino acids

BMI

Body Mass Index

DXA

Dual-Energy x-ray Absorptiometry

EASL

European Association for the Study of the Liver

FFQ

Food Frequency Questionnaire

FGF-21

Fibroblast Growth Factor 21

FLI

Fatty Liver Index

FLiO

Fatty Liver in Obesity

GGT

Gamma-Glutamyl Transferase

GIANT

Genetic Investigation of ANtropometric Traits consortium

GRS

Genetic Risk Score

GWAs

Genome-Wide Associations

$\mathrm{HCC}$

Hepatocellular Carcinoma

HOMA-IR

Homeostasis Model Assessment Insulin Resistance

IQR

Interquartile Ranges

MedDiet Score

Mediterranean diet Score

METs

Metabolic equivalent of the task

MRI

Magnetic Resonance Imaging

NAFLD

Non-alcoholic fatty liver disease

$\mathrm{NASH}$ 
Non-alcoholic steatohepatitis

OWLiver ${ }^{\circledR}$

One Way Liver ${ }^{\circledR}$

PA

Physical activity

SD

Standard Deviations

SNPs

Single Nucleotide Polymorphisms

TG

Triglycerides

TGs

Triacylglycerols

TyG index

Triglycerides and Glucose Index

T2D

Type 2 Diabetes

VAT

Visceral Adipose Tissue

WC

Waist Circumference

\section{Declarations}

Ethics approval and consent to participate The present study was approved by the Research Ethics Committee of the University of Navarra on 24 April 2015 (ref. 54/2015) and has therefore been performed in accordance with the ethical standards laid down in the 1964 Declaration of Helsinki and its latter amendments. All participants gave written informed consent in a clinical interview for their participation in the study.

Consent for publication Not applicable

Availability of data and material The datasets used and/or analysed during the current study are available from the corresponding author on reasonable request.

Competing interest The authors declare no conflict of interest concerning this research.

Funding This research was funded by the Health Department of the Government of Navarra (61/2015), CIBERobn (Physiopathology of Obesity and Nutrition) (CB12/03/3002) and Fundació La Marató de TV3 (201630.10). 
Authors" contributions Conceptualization, N.P.-D.-d.-C., J.I.R.-B., B.A.M.-A., J.I.M., M.E., J.I.H., A.B.-B., F.I.M., J.A.T., I.A., M.A.Z. and J.A.M; methodology, N.P.-D.-d.-C., J.I.R.-B., B.A.M.-A., J.I.M., M.E., J.I.H., A.B.-B., F.I.M., J.A.T., I.A., M.A.Z. and J.A.M.; validation, N.P.-D.-d.-C., J.I.R.-B., M.A.Z. and J.A.M.; formal analysis, N.P.-D.d.-C., J.I.R.-B., M.A.Z. and J.A.M; investigation, N.P.-D.-d.-C., J.I.R.-B., J.A.M. and M.A.Z.; resources, N.P.-D.d.-C., J.I.R.-B., M.A.Z. and J.A.M.; data curation, N.P.-D.-d.-C., J.I.R.-B., M.A.Z. and J.A.M.; writing-original draft preparation, N.P.-D.-d.-C., J.I.R.-B., M.A.Z. and J.A.M.; writing-review and editing, N.P.-D.-d.-C., J.I.R.-B., M.A.Z. and J.A.M.; visualization, N.P.-D.-d.-C., J.I.R.-B., M.A.Z. and J.A.M.; supervision, N.P.-D.-d.-C., J.I.R.-B., M.A.Z. and J.A.M.; project administration, J.A.T., J.I.R.-B., 'M.A.Z. and J.A.M.; funding acquisition, J.A.T., M.A.Z. and J.A.M.; All authors have read and agreed to the published version of the manuscript

Acknowledgements The authors are grateful to the volunteers of the study as well as Veronica Ciaurriz, Amanda Cuevas-Sierra and to the nurses from the departments of Clinical Chemistry, Radiology, Internal Medicine and the Liver Unit of the Clinica Universidad de Navarra for their contribution to FLiO project. The pre-doctoral research grant to Nuria Pérez from the Centre for Nutrition Research of the University of Navarra is gratefully acknowledged. The tractor role from LABORATORIOS CINFA, S.A. and VISCOFAN S.A. for financial support of the Center for Nutrition Research as well as the support from the Government of Navarra are also appreciated. Finally, the authors wish to express their gratitude to the Government of Navarra, CIBERobn and Fundació La Marató de TV3 for the financial support.

\section{References}

1. Trépo E, Valenti L. Update on NAFLD genetics: From new variants to the clinic. J Hepatol. 2020;72:1196-209. doi:10.1016/j.jhep.2020.02.020.

2. Koch LK, Yeh MM. Nonalcoholic fatty liver disease (NAFLD): Diagnosis, pitfalls, and staging. Ann Diagn Pathol. 2018;37:83-90. doi:10.1016/j.anndiagpath.2018.09.009.

3. Mundi MS, Velapati S, Patel J, Kellogg TA, Abu Dayyeh BK, Hurt RT. Evolution of NAFLD and Its Management. Nutr Clin Pract. 2020;35:72-84.

4. Galarregui C, Zulet M, Cantero I, Marín-Alejandre BA, Monreal JI, Elorz M, et al. Interplay of glycemic index, glycemic load, and dietary antioxidant capacity with insulin resistance in subjects with a cardiometabolic risk profile. Int J Mol Sci. 2018;19.

5. Zusi C, Mantovani A, Olivieri F, Morandi A, Corradi M, Miraglia Del Giudice E, et al. Contribution of a genetic risk score to clinical prediction of hepatic steatosis in obese children and adolescents. Dig Liver Dis. 2019;51:1586-92. doi:10.1016/j.dld.2019.05.029.

6. Kupčová V, Fedelešová M, Bulas J, Kozmonová P, Turecký L. Overview of the pathogenesis, genetic, and non-invasive clinical, biochemical, and scoring methods in the assessment of NAFLD. Int J Environ Res Public Health. 2019;16. doi:10.3390/ijerph16193570.

7. Castera L. Diagnosis of non-alcoholic fatty liver disease/non-alcoholic steatohepatitis: Non-invasive tests are enough. Liver Int. 2018;38:67-70. 
8. Petta S, Wong VWS, Cammà C, Hiriart JB, Wong GLH, Vergniol J, et al. Serial combination of noninvasive tools improves the diagnostic accuracy of severe liver fibrosis in patients with NAFLD. Aliment Pharmacol Ther. 2017;46:617-27.

9. Besutti G, Valenti L, Ligabue G, Bassi MC, Pattacini P, Guaraldi G, et al. Accuracy of imaging methods for steatohepatitis diagnosis in non-alcoholic fatty liver disease patients: A systematic review. Liver Int. 2019;39:1521-34.

10. Goni L, Qi L, Cuervo M, Milagro FI, Saris WH, MacDonald IA, et al. Effect of the interaction between diet composition and the PPM1 $\mathrm{K}$ genetic variant on insulin resistance and $\beta$ cell function markers during weight loss: Results from the Nutrient Gene Interactions in Human Obesity: Implications for dietary guidelines (NUGEN. Am J Clin Nutr. 2017;106:902-8.

11. Perakakis N, Polyzos SA, Yazdani A, Sala-Vila A, Kountouras J, Anastasilakis AD, et al. Non-invasive diagnosis of non-alcoholic steatohepatitis and fibrosis with the use of omics and supervised learning: A proof of concept study. Metabolism. 2019;101.

12. Bedogni G, Bellentani S, Miglioli L, Masutti F, Passalacqua M, Castiglione A, et al. The Fatty Liver Index: a simple and accurate predictor of hepatic steatosis in the general population. BMC Gastroenterol. 2006;6:33. doi:10.1186/1471-230X-6-33.

13. Perez-Diaz-Del-Campo N, Abete I, Cantero I, Marin-Alejandre BA, Monreal JI, Elorz M, et al. Association of the SH2B1 RS7359397 gene polymorphism with steatosis severity in subjects with obesity and non-alcoholic fatty liver disease. Nutrients. 2020;12:1-16.

14. León-Mimila P, Vega-Badillo J, Gutiérrez-Vidal R, Villamil-Ramírez H, Villareal-Molina T, LarrietaCarrasco $\mathrm{E}$, et al. A genetic risk score is associated with hepatic triglyceride content and nonalcoholic steatohepatitis in Mexicans with morbid obesity. Exp Mol Pathol. 2015;98:178-83. doi:10.1016/j.yexmp.2015.01.012.

15. Gellert-Kristensen H, Richardson TG, Davey Smith G, Nordestgaard BG, Tybjærg-Hansen A, Stender S. Combined Effect of PNPLA3, TM6SF2, and HSD17B13 Variants on Risk of Cirrhosis and Hepatocellular Carcinoma in the General Population. Hepatology. 2020;72:845-56.

16. Di Costanzo A, Pacifico L, Chiesa C, Perla FM, Ceci F, Angeloni A, et al. Genetic and metabolic predictors of hepatic fat content in a cohort of Italian children with obesity. Pediatr Res. 2019;85:671-7.

17. Pelusi S, Baselli G, Pietrelli A, Dongiovanni P, Donati B, McCain MV, et al. Rare Pathogenic Variants Predispose to Hepatocellular Carcinoma in Nonalcoholic Fatty Liver Disease. Sci Rep. 2019;9:1-10.

18. Loomba R, Schork N, Chen CH, Bettencourt R, Bhatt A, Ang B, et al. Heritability of Hepatic Fibrosis and Steatosis Based on a Prospective Twin Study. Gastroenterology. 2015;149:1784-93. doi:10.1053/j.gastro.2015.08.011.

19. Degasperi E, Galmozzi E, Pelusi S, D’Ambrosio R, Soffredini R, Borghi M, et al. Hepatic Fat-Genetic Risk Score Predicts Hepatocellular Carcinoma in Patients With Cirrhotic HCV Treated With DAAs. Hepatology. 2020;72:1912-23. doi:10.1002/hep.31500. 
20. Goodarzi MO. Genetics of obesity: what genetic association studies have taught us about the biology of obesity and its complications. Lancet Diabetes Endocrinol. 2018;6:223-36. doi:10.1016/S22138587(17)30200-0.

21. Leoni S, Tovoli F, Napoli L, Serio I, Ferri S, Bolondi L. Current guidelines for the management of nonalcoholic fatty liver disease: A systematic review with comparative analysis. World J Gastroenterol. 2018;24:3361-73.

22. Rinella ME, Tacke F, Sanyal AJ, Anstee QM. Report on the AASLD/EASL Joint Workshop on Clinical Trial Endpoints in NAFLD. Hepatology. 2019;70:1424-36.

23. Abenavoli L, Boccuto L, Federico A, Dallio M, Loguercio C, Renzo L, Di, et al. Diet and Non-Alcoholic Fatty Liver Disease: The Mediterranean Way. Int J Environ Res Public Heal Rev. 2019;16:3011.

24. Martinez JA, Navas-Carretero S, Saris WHM, Astrup A. Personalized weight loss strategies - The role of macronutrient distribution. Nature Reviews Endocrinology. 2014;10:749-60. doi:10.1038/nrendo.2014.175.

25. Sheka AC, Adeyi O, Thompson J, Hameed B, Crawford PA, Ikramuddin S. Nonalcoholic Steatohepatitis: A Review. JAMA - J Am Med Assoc. 2020;323:1175-83.

26. Ramos-Lopez O, Cuervo M, Goni L, Milagro Fl, Riezu-Boj Jl, Martinez JA. Modeling of an integrative prototype based on genetic, phenotypic, and environmental information for personalized prescription of energy-restricted diets in overweight/obese subjects. Am J Clin Nutr. 2020;111:459-70.

27. O'Connor D, Pang M, Castelnuovo G, Finlayson G, Blaak E, Gibbons C, et al. A rational review on the effects of sweeteners and sweetness enhancers on appetite, food reward and metabolic/adiposity outcomes in adults. Food Funct. 2021;12:442-65. doi:10.1039/d0fo02424d.

28. González-Muniesa P, Alfredo Martínez J. Precision nutrition and metabolic syndrome management. Nutrients. 2019;11:23-5.

29. San-Cristobal R, Navas-Carretero S, Martínez-González M, Ordovas JM, Martínez JA. Contribution of macronutrients to obesity: implications for precision nutrition. Nat Rev Endocrinol. 2020;16:305-20. doi:10.1038/s41574-020-0346-8.

30. Cantero I, Elorz M, Abete I, Marin BA, Herrero JI, Monreal JI, et al. Ultrasound/elastography techniques, lipidomic and blood markers compared to magnetic resonance imaging in non-alcoholic fatty liver disease adults. Int J Med Sci. 2019;16:75-83.

31. Marin-alejandre BA, Abete I, Cantero I, Monreal JI, Martinez-echeverria A, Uriz-otano JI. The Metabolic and Hepatic Impact of Two Personalized Dietary Strategies in Subjects with Obesity and Nonalcoholic Fatty Liver Disease: The Fatty Liver in Obesity (FLiO) Randomized controlled trial. Nutrients. 2019;11.

32. Marin-Alejandre BA, Cantero I, Perez-Diaz-del-Campo N, Monreal JI, Elorz M, Herrero JI, et al. Effects of two personalized dietary strategies during a 2-year intervention in subjects with nonalcoholic fatty liver disease: a randomized trial. Liver Int. 2021. doi:10.1111/liv.14818.

33. Perez-Diaz-del-Campo N, Marin-Alejandre BA, Cantero I, Monreal JI, Elorz M, Herrero Jl, et al. Differential response to a 6-month energy-restricted treatment depending on SH2B1 rs7359397 
variant in NAFLD subjects: Fatty Liver in Obesity (FLiO) Study. Eur J Nutr. 2021. doi:10.1007/s00394020-02476-x.

34. Sanyal AJ, Brunt EM, Kleiner DE, Kowdley KV, Chalasani N, Lavine JE, et al. Endpoints and clinical trial design for nonalcoholic steatohepatitis. Hepatology. 2011;54:344-53.

35. Chalasani N, Younossi Z, Lavine JE, Charlton M, Cusi K, Rinella M, et al. The diagnosis and management of nonalcoholic fatty liver disease: Practice guidance from the American Association for the Study of Liver Diseases. Hepatology. 2018;67:328-57.

36. Fernández-Ballart JD, Piñol JL, Zazpe I, Corella D, Carrasco P, Toledo E, et al. Relative validity of a semi-quantitative food-frequency questionnaire in an elderly Mediterranean population of Spain. $\mathrm{Br} \mathrm{J}$ Nutr. 2010;103:1808-16.

37. Recaredo G, Marin-Alejandre BA, Cantero I, Monreal JI, Herrero JI, Benito-Boillos A, et al. Association between Different Animal Protein Sources and Liver Status in Obese Subjects with Non-Alcoholic Fatty Liver Disease: Fatty Liver in Obesity (FLiO) Study. Nutrients. 2019;11. doi:10.3390/nu11102359.

38. Martínez-González MA, Buil-Cosiales P, Corella D, Bulló M, Fitó M, Vioque J, et al. Cohort profile: Design and methods of the PREDIMED-Plus randomized trial. Int J Epidemiol. 2019;48:387-80.

39. Galmes-Panades AM, Konieczna J, Abete I, Colom A, Rosique-Esteban N, Zulet MA, et al. Lifestyle factors and visceral adipose tissue: Results from the PREDIMED-PLUS study. PLoS One. 2019;14:115.

40. Elosua R, Garcia M, Aguilar A, Molina L, Covas M-I, Marrugat J. Validation of the Minnesota Leisure Time Spanish Women. Med Sci Sport Exerc. 2000;32:1431-7.

41. Elosua R, Garcia M, Aguilar A, Molina L, Covas MI, Marrugat J. Validation of the Minnesota Leisure Time Physical Activity Questionnaire in Spanish Men. Med Sci Sports Exerc. 1994;32:1431-7.

42. de la Iglesia R, Lopez-Legarrea P, Abete I, Bondia-Pons I, Navas-Carretero S, Forga L, et al. A new dietary strategy for long-term treatment of the metabolic syndrome is compared with the American Heart Association (AHA) guidelines: the MEtabolic Syndrome REduction in NAvarra (RESMENA) project. Br J Nutr. 2014;111:643-52.

43. Navarro-González D, Sánchez-Íñigo L, Pastrana-Delgado J, Fernández-Montero A, Martinez JA. Triglyceride-glucose index (TyG index) in comparison with fasting plasma glucose improved diabetes prediction in patients with normal fasting glucose: The Vascular-Metabolic CUN cohort. Prev Med (Baltim). 2016;86:99-105.

44. Marin-Alejandre BA, Abete I, Cantero I, Riezu-Boj Jl, Milagro FI, Monreal Jl, et al. Association between sleep disturbances and liver status in obese subjects with nonalcoholic fatty liver disease: A comparison with healthy controls. Nutrients. 2019;11:1-16.

45. Lee SS, Park SH. Radiologic evaluation of nonalcoholic fatty liver disease. World J Gastroenterol. 2014;20:7392-402.

46. Cassinotto $C$, Boursier J, de Lédinghen V, Lebigot J, Lapuyade $B$, Cales $P$, et al. Liver stiffness in nonalcoholic fatty liver disease: A comparison of supersonic shear imaging, FibroScan, and ARFI 
with liver biopsy. Hepatology. 2016;63:1817-27.

47. Bril F, Millán L, Kalavalapalli S, McPhaul MJ, Caulfield MP, Martinez-Arranz I, et al. Use of a metabolomic approach to non-invasively diagnose non-alcoholic fatty liver disease in patients with type 2 diabetes mellitus. Diabetes, Obes Metab. 2018;20:1702-9.

48. Ramos-Lopez O, Milagro FI, Allayee H, Chmurzynska A, Choi MS, Curi R, et al. Guide for current nutrigenetic, nutrigenomic, and nutriepigenetic approaches for precision nutrition involving the prevention and management of chronic diseases associated with obesity. J Nutrigenet Nutrigenomics. 2017;10:43-62. doi:10.1159/000477729.

49. Heianza Y, Ma W, Huang T, Wang T, Zheng Y, Smith SR, et al. Macronutrient intake-associated FGF21 genotype modifies effects of weight-loss diets on 2-year changes of central adiposity and body composition: The POUNDS lost trial. Diabetes Care. 2016;39:1909-14.

50. Goni L, Cuervo M, Milagro FI, Martínez JA. Gene-gene interplay and gene-diet interactions involving the MTNR1B rs10830963 variant with body weight loss. J Nutrigenet Nutrigenomics. 2015;7:232-42.

51. Guo F, Zhou Y, Song H, Zhao J, Shen H, Zhao B, et al. Next generation sequencing of SNPs using the HID-Ion AmpliSeq ${ }^{\mathrm{TM}}$ Identity Panel on the Ion Torrent PGM ${ }^{\mathrm{TM}}$ platform. Forensic Sci Int Genet. 2016;25:73-84. doi:10.1016/j.fsigen.2016.07.021.

52. Ramos-Lopez O, Riezu-Boj JI, Milagro Fl, Cuervo M, Goni L, Martinez JA. Prediction of blood lipid phenotypes using obesity-related genetic polymorphisms and lifestyle data in subjects with excessive body weight. Int J Genomics. 2018;2018. doi:10.1155/2018/4283078.

53. Ramos-Lopez O, Riezu-Boj Jl, Milagro Fl, Cuervo M, Goni L, Alfredo Martinez J. Models integrating genetic and lifestyle interactions on two adiposity phenotypes for personalized prescription of energy-restricted diets with different macronutrient distribution. Front Genet. 2019;10 JUL:1-11.

54. Cuevas-Sierra A, Riezu-Boj JI, Guruceaga E, Milagro FI, Martínez JA. Sex-specific associations between gut prevotellaceae and host genetics on adiposity. Microorganisms. 2020;8:1-19.

55. Katsiki N, Gastaldelli A, Mikhailidis DP. Predictive models with the use of omics and supervised machine learning to diagnose non-alcoholic fatty liver disease: A "non-invasive alternative" to liver biopsy? Metabolism: Clinical and Experimental. 2019;101.

56. Bessone F, Razori MV, Roma MG. Molecular pathways of nonalcoholic fatty liver disease development and progression. Cell Mol Life Sci. 2019;76:99-128. doi:10.1007/s00018-018-2947-0.

57. Rui L. SH2B1 regulation of energy balance, body weight, and glucose metabolism. World J Diabetes. 2014;5:511.

58. Ferguson LR, De Caterina R, Görman U, Allayee H, Kohlmeier M, Prasad C, et al. Guide and Position of the International Society of Nutrigenetics/Nutrigenomics on Personalised Nutrition: Part 1 - Fields of Precision Nutrition. J Nutrigenet Nutrigenomics. 2016;9:12-27.

59. Anstee QM, Darlay R, Cockell S, Meroni M, Govaere O, Tiniakos D, et al. Genome-wide association study of non-alcoholic fatty liver and steatohepatitis in a histologically characterised cohort】. J Hepatol. 2020;73:505-15. 
60. Younossi Z, Anstee QM, Marietti M, Hardy T, Henry L, Eslam M, et al. Global burden of NAFLD and NASH: Trends, predictions, risk factors and prevention. Nat Rev Gastroenterol Hepatol. 2018;15:1120. doi:10.1038/nrgastro.2017.109.

61. Younossi Z, Tacke F, Arrese M, Chander Sharma B, Mostafa I, Bugianesi E, et al. Global Perspectives on Nonalcoholic Fatty Liver Disease and Nonalcoholic Steatohepatitis. Hepatology. 2019;69:267282. doi:10.1002/hep.30251.

62. Kabisch S, Bäther S, Dambeck U, Kemper M, Gerbracht C, Honsek C, et al. Liver fat scores moderately reflect interventional changes in liver fat content by a low-fat diet but not by a low-carb diet. Nutrients. 2018;10.

63. Calori G, Lattuada G, Ragogna F, Garancini MP, Crosignani P, Villa M, et al. Fatty liver index and mortality: The cremona study in the 15th year of follow-up. Hepatology. 2011;54:145-52.

64. Motamed N, Faraji AH, Khonsari MR, Maadi M, Tameshkel FS, Keyvani H, et al. Fatty liver index (FLI) and prediction of new cases of non-alcoholic fatty liver disease: A population-based study of northern Iran. Clin Nutr. 2020;39:468-74. doi:10.1016/j.clnu.2019.02.024.

65. Li C, Guo P, Zhang R, Zhang M, Li Y, Huang M, et al. Both WHR and FLI as Better Algorithms for Both Lean and Overweight/Obese NAFLD in a Chinese Population. J Clin Gastroenterol. 2019;53:E253-60.

66. Karlas T, Petroff D, Garnov N, Böhm S, Tenckhoff H, Wittekind C, et al. Non-invasive assessment of hepatic steatosis in patients with NAFLD using controlled attenuation parameter and $1 \mathrm{H}-\mathrm{MR}$ spectroscopy. PLoS One. 2014;9.

67. Mayo R, Crespo J, Martínez-Arranz I, Banales JM, Arias M, Mincholé I, et al. Metabolomic-based noninvasive serum test to diagnose nonalcoholic steatohepatitis: Results from discovery and validation cohorts. Hepatol Commun. 2018;2:807-20.

68. Matsuo T, Nakata Y, Katayama Y, lemitsu M, Maeda S, Okura T, et al. PPARG genotype accounts for part of individual variation in body weight reduction in response to calorie restriction. Obesity. 2009;17:1924-31. doi:10.1038/oby.2009.199.

69. Qi L, Kraft P, Hunter DJ, Hu FB. The common obesity variant near MC4R gene is associated with higher intakes of total energy and dietary fat, weight change and diabetes risk in women. Hum Mol Genet. 2008;17:3502-8.

70. Sanghera DK, Bejar C, Sharma S, Gupta R, Blackett PR. Obesity genetics and cardiometabolic health: Potential for risk prediction. Diabetes Obes Metab. 2019;21:1088-100.

71. Locke AE, Kahali B, Berndt SI, Justice AE, Pers TH, Day FR, et al. Genetic studies of body mass index yield new insights for obesity biology. Nature. 2015;518:197-206. doi:10.1038/nature14177.

72. Celis-Morales CA, Lyall DM, Gray SR, Steell L, Anderson J, Iliodromiti S, et al. Dietary fat and total energy intake modifies the association of genetic profile risk score on obesity: Evidence from 48170 UK Biobank participants. Int J Obes. 2017;41:1761-8. doi:10.1038/ijo.2017.169.

73. Eslam M, Newsome PN, Sarin SK, Anstee QM, Targher G, Romero-Gomez M, et al. A new definition for metabolic dysfunction-associated fatty liver disease: An international expert consensus statement. J Hepatol. 2020;73:202-9. doi:10.1016/j.jhep.2020.03.039. 
74. Moonesinghe R, Liu T, Khoury MJ. Evaluation of the discriminative accuracy of genomic profiling in the prediction of common complex diseases. Eur J Hum Genet. 2010;18:485-9. doi:10.1038/ejhg.2009.209.

75. Pierantonelli I, Svegliati-Baroni G. Nonalcoholic Fatty Liver Disease: Basic Pathogenetic Mechanisms in the Progression from NAFLD to NASH. Transplantation. 2019;103:E1-13.

76. Tucker B, Li H, Long X, Rye KA, Ong KL. Fibroblast growth factor 21 in non-alcoholic fatty liver disease. Metabolism: Clinical and Experimental. 2019;101.

77. Goni L, Riezu-Boj Jl, Milagro FI, Corrales FJ, Ortiz L, Cuervo M, et al. Interaction between an ADCY3 Genetic Variant and TwoWeight-Lowering Diets Affecting Body Fatness and Body Composition Outcomes Depending on Macronutrient Distribution: A Randomized Trial Leticia. Nutrients. 2018;10:1-10.

78. Cantero I, Abete I, Monreal JI, Martinez JA, Zulet MA. Fruit fiber consumption specifically improves liver health status in obese subjects under energy restriction. Nutrients. 2017;9.

79. Bullón-Vela V, Abete I, Tur JA, Pintó X, Corbella E, Martínez-González MA, et al. Influence of lifestyle factors and staple foods from the Mediterranean diet on non-alcoholic fatty liver disease among older individuals with metabolic syndrome features. Nutrition. 2020;71. doi:10.1016/j.nut.2019.110620.

80. Qi Q, Chu AY, Kang JH, Jensen MK, Curhan GC, Pasquale LR, et al. Sugar-Sweetened Beverages and Genetic Risk of Obesity. N Engl J Med. 2012;367:1387-96.

81. Goni L, García-granero M, Milagro Fl, Cuervo M. Phenotype and genotype predictors of BMI variability among European adults. Nutr Diabetes. 2018;:1-8. doi:10.1038/s41387-018-0041-1.

82. Rask-Andersen M, Karlsson T, Ek WE, Johansson Å. Gene-environment interaction study for BMI reveals interactions between genetic factors and physical activity, alcohol consumption and socioeconomic status. PLoS Genet. 2017;13:1-20.

83. Mangum BP, Mangum T. Gene-Environment Interactions and the Genetic Epidemiology of Obesity: Correlates for Preventative Medicine. SSRN Electron J. 2018;1:25-8.

84. Qi Q, Downer MK, Kilpelainen TO, Taal HR, Barton SJ, Ntalla I, et al. Dietary Intake, FTO genetic variants, and adiposity: A combined analysis of over 16,000 children and adolescents. Diabetes. 2015;64:2467-76. doi:10.2337/db14-1629.

85. Bergeron N, Chiu S, Williams PT, King M, Krauss S. RM. Effects of red meat, white meat, and nonmeat protein sources on atherogenic lipoprotein measures in the context of low compared with high saturated fat intake: a randomized controlled trial. Am J Clin Nutr. 2019;110:24-33.

86. Navas-Carretero S, San-Cristobal R, Livingstone KM, Celis-Morales C, Marsaux CF, Macready AL, et al. Higher vegetable protein consumption, assessed by an isoenergetic macronutrient exchange model, is associated with a lower presence of overweight and obesity in the web-based Food4me European study. Int J Food Sci Nutr. 2019;70:240-53.

87. Galarregui C, Cantero I, Marin-Alejandre BA, Monreal JI, Elorz M, Benito-Boillos A, et al. Dietary intake of specific amino acids and liver status in subjects with nonalcoholic fatty liver disease: fatty liver in 
obesity (FLiO) study. Eur J Nutr. 2020. doi:10.1007/s00394-020-02370-6.

88. Newgard CB. Interplay between lipids and branched-chain amino acids in development of insulin resistance. Cell Metab. 2012;15:606-14. doi:10.1016/j.cmet.2012.01.024.

89. Alsulami S, Aji AS, Ariyasra U, Sari SR, Tasrif N, Yani FF, et al. Interaction between the genetic risk score and dietary protein intake on cardiometabolic traits in Southeast Asian. Genes Nutr. 2020;15. doi:10.1186/s12263-020-00678-w.

90. De Chiara F, Checcllo CU, Azcón JR. High protein diet and metabolic plasticity in non-alcoholic fatty liver disease: Myths and truths. Nutrients. 2019;11.

91. Honda T, Ishigami M, Luo F, Lingyun M, Ishizu Y, Kuzuya T, et al. Branched-chain amino acids alleviate hepatic steatosis and liver injury in choline-deficient high-fat diet induced NASH mice. Metabolism. 2017;69:177-87. doi:10.1016/j.metabol.2016.12.013.

92. Takegoshi K, Honda M, Okada H, Takabatake R, Matsuzawa-Nagata N, Campbell JS, et al. Branchedchain amino acids prevent hepatic fibrosis and development of hepatocellular carcinoma in a nonalcoholic steatohepatitis mouse model. Oncotarget. 2017;8:18191-205.

93. Wray NR, Lee SH, Mehta D, Vinkhuyzen AAE, Dudbridge F, Middeldorp CM. Research Review: Polygenic methods and their application to psychiatric traits. J Child Psychol Psychiatry Allied Discip. 2014;55:1068-87.

\section{Figures}



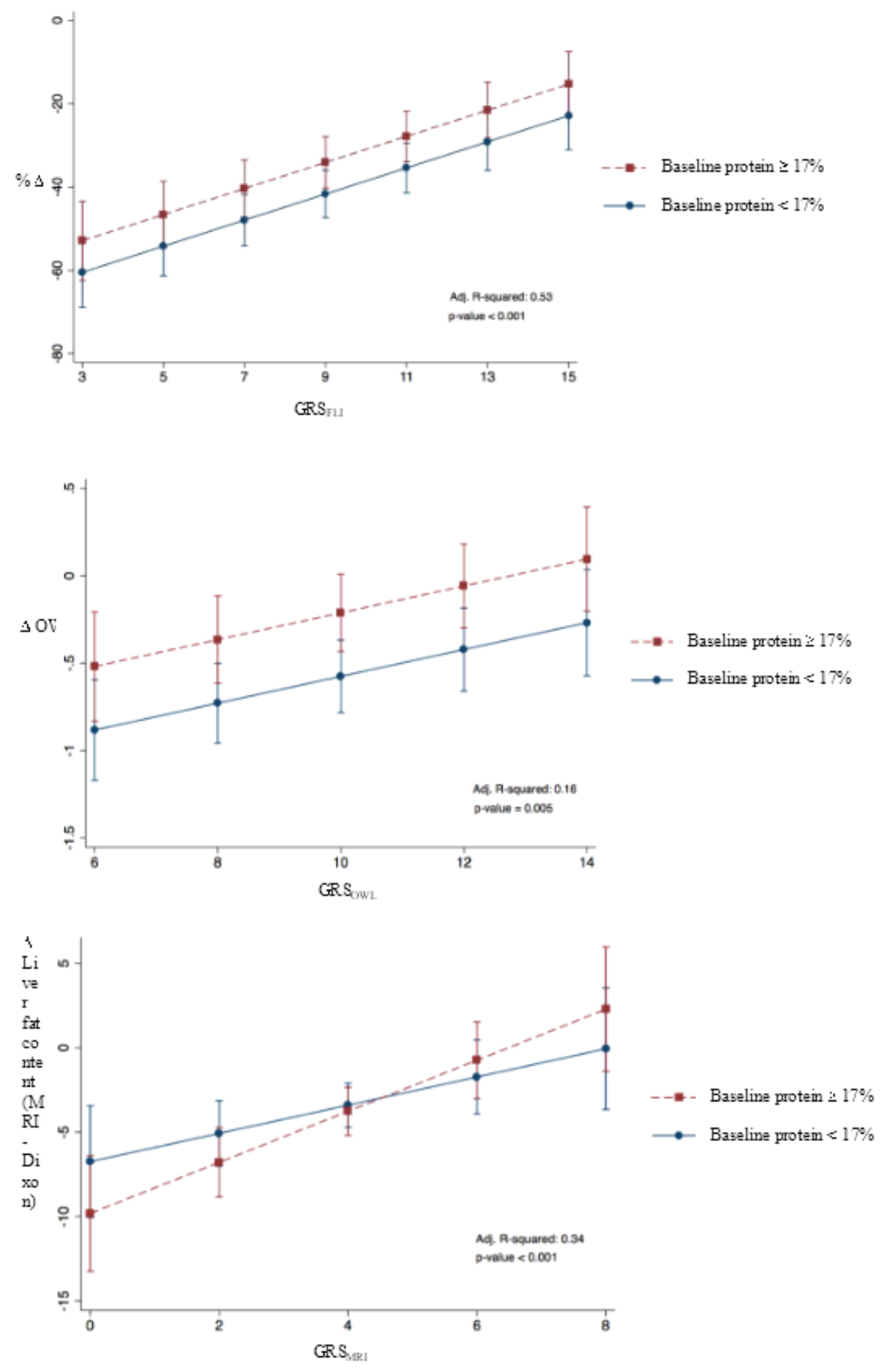

\section{Figure 1}

Effect of the changes in a) \% FLI and GRSFLI, B) OWLiver ${ }^{\circledR}$-test and GRSOWL and C) MRI and GRSMRI and baseline protein after 6-months nutritional treatment. Baseline protein was dychotomized according to median.

\section{Supplementary Files}


This is a list of supplementary files associated with this preprint. Click to download.

- Supplementarymaterial.pdf 